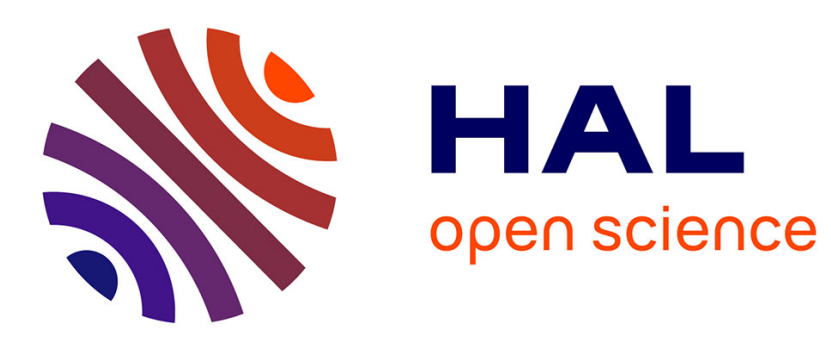

\title{
Identification of block-oriented systems in presence of nonparametric input nonlinearities of switch and backlash types
}

Youssef Rochdi, Fouad Giri, Jean-Baptiste Gning, Fatima Zara Chaoui

\section{- To cite this version:}

Youssef Rochdi, Fouad Giri, Jean-Baptiste Gning, Fatima Zara Chaoui. Identification of block-oriented systems in presence of nonparametric input nonlinearities of switch and backlash types. Automatica, 2010, 46 (5), pp.864-877. 10.1016/j.automatica.2010.02.020 . hal-01059884

\section{HAL Id: hal-01059884 \\ https://hal.science/hal-01059884}

Submitted on 9 Sep 2014

HAL is a multi-disciplinary open access archive for the deposit and dissemination of scientific research documents, whether they are published or not. The documents may come from teaching and research institutions in France or abroad, or from public or private research centers.
L'archive ouverte pluridisciplinaire HAL, est destinée au dépôt et à la diffusion de documents scientifiques de niveau recherche, publiés ou non, émanant des établissements d'enseignement et de recherche français ou étrangers, des laboratoires publics ou privés. 


\title{
Identification of block-oriented systems in the presence of nonparametric input nonlinearities of switch and backlash types
}

\author{
Y. Rochdi ${ }^{a}$, F. Giri ${ }^{\text {b,* }}$, J.B. Gning ${ }^{\text {b }}$, F.Z. Chaoui ${ }^{c}$ \\ a University Cadi Ayyad, Marrakech, Morocco \\ ${ }^{\mathrm{b}}$ GREYC Lab, University of Caen Basse-Normandie, Caen, France \\ ${ }^{\mathrm{C}}$ ENSET, University of Rabat, Morocco
}

Keywords:

System identification

Hammerstein-like systems

Nonparametric nonlinearities

Switch

Backlash

Experiment design

Persistent excitation

Estimator consistency

\begin{abstract}
A B S T R A C T
The problem of identifying Hammerstein-like systems containing dynamic nonlinearities, of the switch or backlash types, is considered. Interestingly, the nonlinearity borders are nonparametric borders (i.e. of unknown structure) and so are allowed to be noninvertible and cross each other. A semi-parametric identification approach is developed to estimate the linear subsystem parameters and $m$ points on both nonlinearity borders. It relies on two main experiments designed so that during each one, the focus is on one lateral border exciting $m$ specific points. Doing so, the initial nonparametric identification problem is decomposed into two simpler problems involving static parametric nonlinearities. The new problems are dealt with independently using least squares type estimators. It is formally shown that the experiments generate persistently exciting signals ensuring the consistency of all involved parameter estimators.
\end{abstract}

\section{Introduction}

Nonlinear block-oriented models have proved to be useful in capturing nonlinear behaviors of many physical systems e.g. Eskinat, Johnson, and Luyben (1991), Greblicki and Pawlak (2008), Kim and Konstantinou (2001) and Palanthandalam-Madapusi, Bernstein, and Ridley (2006). They are composed of linear dynamic blocks and nonlinear static operators connected together in several ways. The most well known of such structures is the Hammerstein model focused on in this paper (Fig. 1). Though several identification methods have been developed for this class of models, most of the solutions were developed supposing the nonlinear operator $F[$. $]$ to be static. In such a case, one has $u=F[v] \Leftrightarrow u(t)=$ $\varphi(v(t))$ for some function $\varphi($.$) ; see e.g. Hasiewicz, Sliwinski, and$ Mzyk (2008) and the references listed therein. The proposed solutions can be classed into two categories depending on the nature of the function $\varphi($.). The first one includes methods addressing the

\footnotetext{
The material in this paper was not presented at any conference. This paper was recommended for publication in revised form by Associate Editor Johan Schoukens under the direction of Editor Torsten Söderström.

* Corresponding author. Tel.: +33 231567287; fax: +33 231567340.

E-mail addresses: fouad.giri@unicaen.fr, fouadgiri@yahoo.fr (F. Giri).
}

case of parametric nonlinearities i.e. $\varphi(v)=\varphi_{\theta}(v)$. Then, the identification purpose is to estimate all unknown parameters, those of the linear subsystem and those of the nonlinearity. The function $\varphi_{\theta}(v)$ may be linear in the unknown coefficient vector $\theta$ (Bai $\&$ Li, 2004; Narendra \& Gallman, 1966; Stoica, 1981; Stoica \& Söderström, 1982) or nonlinear (Bai, 2002; Giri, Chaoui, \& Rochdi, 2004; Voros, 1997 and $\mathrm{Gu}$, Bao, \& Lang, 1988). In the case of nonparametric nonlinearity, the aim is to estimate the linear subsystem parameters as well as a finite number of points $\left(v_{i}, \varphi\left(v_{i}\right)\right)(i=1, \ldots, m)$ of the nonlinearity characteristic, (Chaoui, Giri, Rochdi, Haloua, \& Naitali, 2005; Giri, Chaoui, \& Rochdi, 2001; Greblicki \& Pawlak, 2008, 1994; Hasiewicz \& Mzyk, 2009).

The identification of Hammerstein-like ${ }^{1}$ systems with dynamic nonlinearities is a more challenging problem. In this work, the focus is made on backlash and switch operators. These behaviors characterize many physical systems, especially mechanical equipments involving Coulomb's friction (switch) or gears (backlash).

\footnotetext{
1 The Hammerstein model was introduced in 1930 by the German mathematician Hammerstein (1930). It is composed of a 'static' nonlinear element followed in series with an integral operator. In the present work, the model has the same structure as the Hammerstein model but it involves dynamic nonlinearities. Then, it is referred to "Hammerstein-like".
} 
For instance, it is well known that transmission systems cannot work without backlash i.e. the clearance between mating components, sometimes also called lash or play. In the discrete-time context, the switch and backlash behaviors can be described by a first-order difference equation of the form: $u=F[v] \Leftrightarrow u(t)=$ $\varphi(u(t-1), v(t))$. Accordingly, the value $u(t)$ of the inner signal at a given time is not uniquely determined by the value $v(t)$ of the input at the same instant. The function $\varphi(.,$.$) is such that$ the working point $(v(t), u(t))$ only moves along two fixed curves, $\left(v, C_{a}(v)\right)$ and $\left(v, C_{d}(v)\right)$, and (in the backlash case) on horizontal lines connecting these curves (Figs. 2a-2b). As the movement takes place on $\left(v, C_{a}(v)\right)$ when the input signal $v(t)$ increases and on $\left.\left(v, C_{d}(v)\right)\right)$ when $v(t)$ decreases, these curves are referred to 'ascendent' and 'descendent' borders. Few solutions are by now available for Hammerstein-like system identification in the presence of switch and backlash elements (Bai, 2002; Chaoui et al., 2005; Giri, Rochdi, Chaoui, \& Brouri, 2008). Furthermore, the existing solutions have focused on nonlinearities bordered by straight lines. The identification problem has been dealt with using separable nonlinear least squares (Bai, 2002), the bounded error method (Cerone \& Regruto, 2007) or periodic excitation based approach (Giri, Rochdi, Chaoui et al., 2008). The last method has recently been adapted to backlash elements with polynomial borders (Giri, Rochdi, Elayan, Brouri, \& Chaoui, 2008). In this study, we develop a quite different and more powerful identification approach to cope with general switch and backlash nonlinearities (Figs. 2a-2b). The originality lies in the fact that the nonlinearity ascendent and descendent borders, $C_{a}$ and $C_{d}$, are not necessarily straight lines. This phenomenon does exist in practice as shown by Fig. 3 . There, the leaf-spring and Coulomb friction coefficients are let to be what they really are i.e. nonlinear functions of the position $x$. This leads to the switch behavior with nonstraight borders (Fig. 3c). More interestingly, the present study allows the borders to be nonparametric, noninvertible, nonsmooth and crossed by each other (Fig. 4). Allowing borders' crossing constitutes a quite useful feature of the present study. Actually, any identification method conceived for Hammerstein-like systems with switch/backlash nonlinearities must be able to work well in the presence of static nonlinearities. Obviously, static nonlinearities can simply be viewed as dynamic nonlinearities with superposed (ascendent and descendent) borders. But, superposed curves are crossing at an infinite number of points. On the other hand, it is useful to account for nonmonotonic borders because this may arise when a component gets defective modifying the operation mode of the system it belongs to. Then, an identification approach that is able to identify such behavior can be used in fault detection and diagnostic whenever switch/backlash nonlinearities are involved. The identification purpose is the determination of the (parametric) linear subsystem model as well as $m$ points on each border of the (nonparametric) nonlinearity. The abscissas of these points are arbitrarily selected in a given working interval, denoted $\left[\begin{array}{ll}v_{m} & v_{M}\end{array}\right]$ (with $-\infty<v_{m}<v_{M}<+\infty$ ). Our identification method consists of two independent, but structurally-symmetric, identification schemes. The first one, called descendent identification scheme (DIS), determines $m$ points located on the descendent border $C_{d}$ as well as the parameters of the linear subsystem. The second, referred to ascendent identification scheme (AIS), determines $m$ points located on the ascendent border $C_{a}$ and the parameters of the linear subsystem. The two identification schemes operate independently and can be used separately. Each scheme relies on one major experiment designed so that, within the created operational conditions, the considered nonparametric dynamic nonlinearity can be assimilated (with no error) to a parametric static nonlinearity reducing considerably the complexity of the identification problem. The key idea is to use pulse-type periodic input signals so that only the points of interest are excited on each border. Two fully parameterized identification problems are thus got and dealt with separately, using least

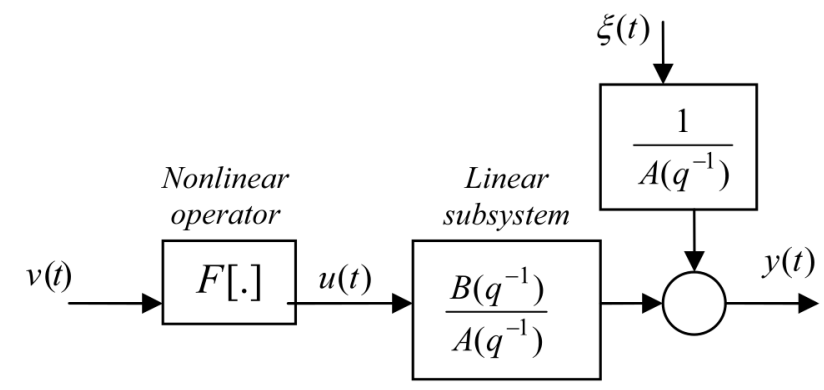

Fig. 1. Hammerstein-like model.

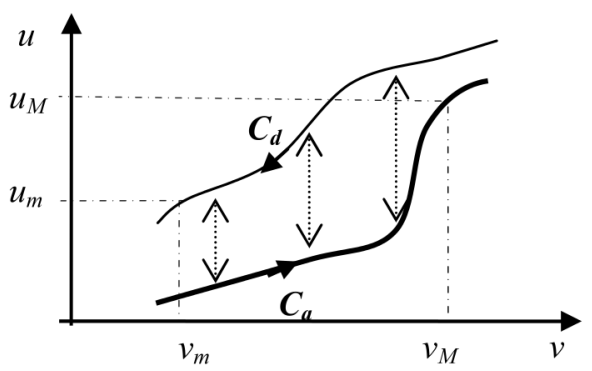

Fig. 2a. General switch nonlinearity.

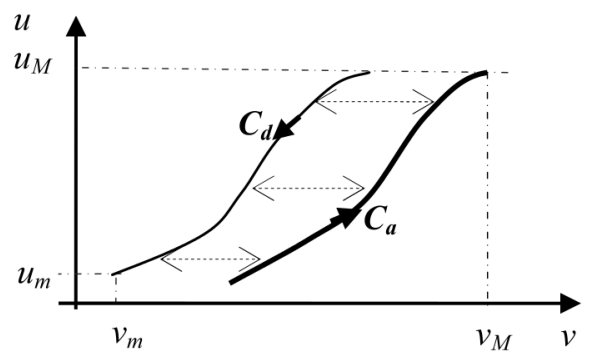

Fig. 2b. General backlash nonlinearity.

squares type estimators. The pulse nature of the input signals is formally shown to provide these estimators with persistent excitation guaranteeing their consistency. The paper is organized as follows: the identification problem is formulated in Section 2; in Section 3, we synthesize the descendent identification scheme and analyze its consistency; the ascendent identification scheme is briefly described in Section 4.

\section{Identification problem statement}

\subsection{Class of identified systems}

We are interested in discrete-time systems that can be described by the Hammerstein-like model (Fig. 1):

$$
\begin{aligned}
& A\left(q^{-1}\right) y(t)=B\left(q^{-1}\right) u(t)+\xi(t) \quad \text { with } u=F[v] \\
& A\left(q^{-1}\right)=1+a_{1} q^{-1}+\cdots+a_{n} q^{-n} \\
& B\left(q^{-1}\right)=b_{1} q^{-1}+\cdots+b_{n} q^{-n}
\end{aligned}
$$

where $v(t)$ and $y(t)$ designate the system input and output; $u(t)$ is an internal signal inaccessible to measurement; $\{\xi(t)\}$ is a zeromean stationary sequence of independent equally distributed random variables. The linear subsystem is stable, controllable and of known order $n$. Controllability of $(2.1)$ is ensured if $A\left(q^{-1}\right)$ and $B\left(q^{-1}\right)$ are coprime; this will be used to establish persistent excitation (Appendix). The nonlinear operator $F[$.] is either a switch or backlash element characterized by its descendent and ascendent lateral borders $\left(v, C_{d}(v)\right)$ and $\left(v, C_{a}(v)\right)$, respectively. These 
a

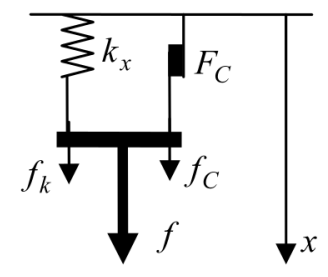

$\mathrm{b}$

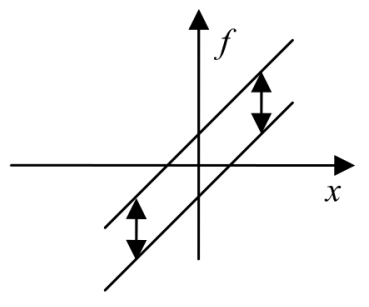

$$
\begin{aligned}
f & =f_{C}+f_{k} \\
& =F_{C} \operatorname{sign}(\dot{x})+k_{x} x
\end{aligned}
$$

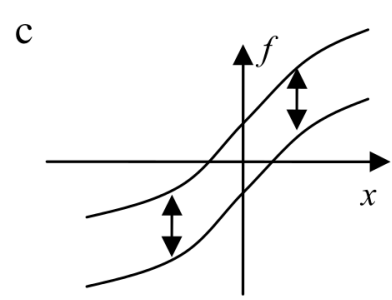

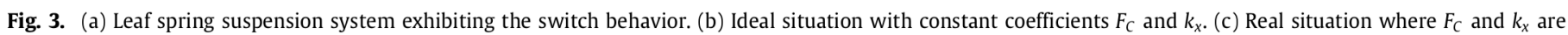
dependent on $x$.

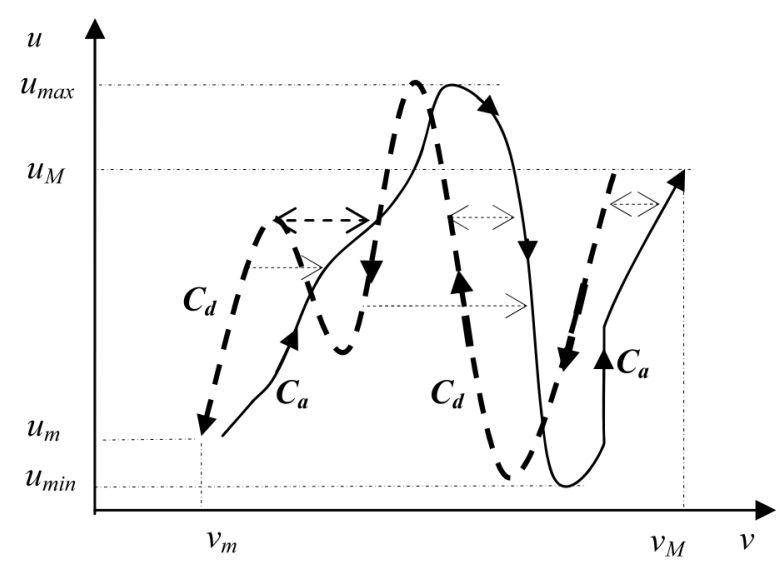

Fig. 4. Backlash nonlinearity with crossing borders.

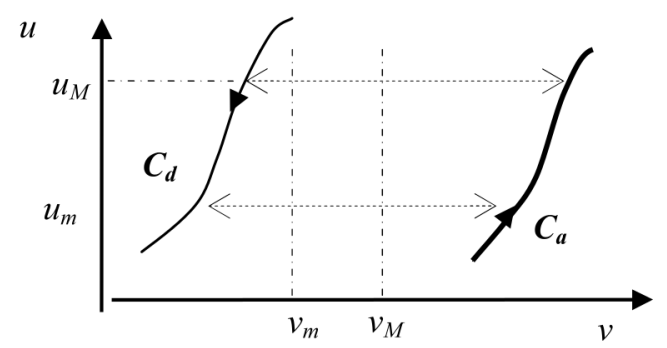

Fig. 5. Example of unsuitable choices of the working interval $\left[\begin{array}{ll}v_{m} & v_{M}\end{array}\right]$ when backlash nonlinearities are considered. In this example, none of the borders can be identified as a constant signal $u(t)$ is rapidly obtained when $v(t)$ spans $\left[v_{m} v_{M}\right]$.

borders assume no particular structure (unlike in Bai (2002), Giri, Rochdi, Elayan et al. (2008) and Chaoui et al. (2005)) and we seek their identification within a given working interval $\left[\begin{array}{ll}v_{m} & v_{M}\end{array}\right]$. This may be arbitrarily chosen in the case of switch nonlinearities. When a backlash element is concerned, the working interval must be sufficiently large to make possible the identifiability of both borders. This point will be made precise latter. Let us first model the switch nonlinearity.

Switch operator modeling. In this case, the working point $(v(t), u(t))$ moves either on $C_{d}(v)$ or $C_{a}(v)$, but never outside. It simply jumps from one border to the other each time the sign of the increment $(v(t)-v(t-1))$ changes. Analytically, a switch element $u=F[v]$ is simply described by the following expression:

$u(t)= \begin{cases}C_{a}(v(t)) & \text { if } v(t)>v(t-1) \\ u(t-1) & \text { if } v(t)=v(t-1) \\ C_{d}(v(t)) & \text { if } v(t)<v(t-1) .\end{cases}$

Backlash operator modeling. In the backlash case, the working point $(v(t), u(t))$ moves not only along the lateral borders but also on horizontal lines connecting these borders. To make clear the operation mode, suppose that the working point $(v(t), u(t))\left(v_{m} \leq\right.$ $\left.v(t) \leq v_{M}\right)$ is currently moving along the border $C_{a}($.$) , which$ means that the input signal $v(t)$ is increasing. If $v(t)$ changes it variation sense at a time $t_{0}$ then the working point will leave the current border $\left(C_{a}().\right)$ and moves horizontally towards the opposite border $C_{d}($.$) . If the input keeps decreasing a sufficiently long$ time, the working point $(v(t), u(t))$ should get to the opposite bor$\operatorname{der} C_{d}($.$) and moves along it. For the point (v(t), u(t))$ to actually reach the border $C_{d}($.$) , the working interval must be sufficiently$ wide as this is illustrated by Figs. $2 \mathrm{~b}$ and 4 . In such cases, a full exhibition of the backlash behavior is ensured whenever the input signal $v(t)$ fully spans the interval $\left[\begin{array}{ll}v_{m} & v_{M}\end{array}\right]$ several times in both senses. Then, the trajectory of the working point $(v(t), u(t))$ is a closed (and connected) cycle including the lateral branches $\left\{\left(v, C_{d}(v)\right), v_{m} \leq v \leq v_{M}\right\}$ and $\left\{\left(v, C_{a}(v)\right), v_{m} \leq v \leq v_{M}\right\}$ as well as the top and bottom horizontal straight lines connecting the above branches (Fig. 7). The opposite case (i.e. not sufficiently wide $\left[\begin{array}{ll}v_{m} & v_{M}\end{array}\right]$ ) is illustrated by Fig. 5 (see also Remark 1, Part b). To formalize the wideness requirement, we need the following notations:

$$
\begin{aligned}
& u_{\min }=\inf _{v_{m} \leq v \leq v_{M}}\left(C_{d}(v), C_{a}(v)\right) \\
& u_{\max }=\sup _{v_{m} \leq v \leq v_{M}}\left(C_{d}(v), C_{a}(v)\right) \\
& C_{a}\left(v_{0}^{-}\right)=\lim _{\substack{v \rightarrow v_{0} \\
v<v_{0}}} C_{a}(v) \text { and } C_{a}\left(v_{0}^{+}\right)=\lim _{\substack{v \rightarrow v_{0} \\
v>v_{0}}} C_{a}(v)
\end{aligned}
$$

$C_{d}\left(v_{0}^{-}\right)$and $C_{d}\left(v_{0}^{+}\right)$are similarly defined. It is clear that $C_{a}\left(v_{0}^{-}\right)=$ $C_{a}\left(v_{0}^{+}\right)=C_{a}\left(v_{0}\right)\left(\right.$ resp. $\left.C_{d}\left(v_{0}^{-}\right)=C_{d}\left(v_{0}^{+}\right)=C_{d}\left(v_{0}\right)\right)$ whenever $C_{a}$ (resp. $C_{d}$ ) is continuous at $v_{0}$. Introduce also the definitions:

$C_{d}^{-1}(u) \stackrel{\text { def }}{=}\left\{v / \min \left(C_{d}\left(v^{-}\right), C_{d}\left(v^{+}\right)\right) \leq u \leq \max \left(C_{d}\left(v^{-}\right), C_{d}\left(v^{+}\right)\right)\right\} ;$ $C_{a}^{-1}(u) \stackrel{\text { def }}{=}\left\{v / \min \left(C_{a}\left(v^{-}\right), C_{a}\left(v^{+}\right)\right) \leq u \leq \max \left(C_{a}\left(v^{-}\right), C_{d}\left(v^{+}\right)\right)\right\}$.

The distinction between $v^{-}$and $v^{+}$in the above expressions is considered to deal with noncontinuous functions $C_{a}$ and $C_{d}$. In the case of continuous borders, the above definitions simplify to the usual expressions: $C_{d}^{-1}(u)=\left\{v / u=C_{d}(v)\right\}$ and $C_{a}^{-1}(u)=$ $\left\{v / u=C_{a}(v)\right\}$.

Assumption 1 (Backlash Only). The working interval $\left[v_{m} v_{M}\right]$ must be wide enough so that, when the input signal spans this interval several times, in both senses, the measured output does not remain constant (up to noise effect) after a transient period. This statement is formalized as follows:

(a) $\forall u \in\left[u_{\min }, u_{\max }\right], \exists v \in\left[\max C_{d}^{-1}(u) v_{M}\right]$ such that:

$\min \left(C_{a}\left(v^{-}\right), C_{a}\left(v^{+}\right)\right) \leq u \leq \max \left(C_{a}\left(v^{-}\right), C_{a}\left(v^{+}\right)\right)$.

The above expression obviously reduces to $u=C_{a}(v)$ when $C_{a}$ is continuous at $v$. 
(b) $\forall u \in\left[u_{\min }, u_{\max }\right], \exists v \in\left[v_{m} \quad \min C_{a}^{-1}(u)\right]$ such that:

$\min \left(C_{d}\left(v^{-}\right), C_{d}\left(v^{+}\right)\right) \leq u \leq \max \left(C_{d}\left(v^{-}\right), C_{d}\left(v^{+}\right)\right)$.

Of course, the above expression simply reduces to $u=C_{d}(v)$ if $C_{d}$ is continuous at $v$.

Under such assumption, a backlash element $u=F[v]$ undergoes within the working interval $\left[v_{m} v_{M}\right]$ the following equation:

$u(t)= \begin{cases}C_{d}(v(t)) & \text { if } v(t) \leq \max \left\{\left[v_{m} v(t-1)\right] \cap C_{d}^{-1}(u(t-1))\right\} \\ C_{a}(v(t)) & \text { if } v(t) \geq \min \left\{\left[v(t-1) v_{M}\right] \cap C_{a}^{-1}(u(t-1))\right\}(2.4 \\ u(t-1) & \text { otherwise. }\end{cases}$

The above system description is completed with the following assumption:

Assumption 2 (Switch and Backlash). The functions $C_{a}$ and $C_{d}$ are bounded in the interval $\left[v_{m}, v_{M}\right]$ and satisfy the property:

$u_{m} \stackrel{\text { def }}{=} C_{d}\left(v_{m}\right) \neq C_{a}\left(v_{M}\right) \stackrel{\text { def }}{=} u_{M}$.

Property (2.5) simply ensures that, when $v(t)$ jumps from $v_{m}$ to $v_{M}$ (or vice versa) the internal signal $u(t)=F[v](t)$ changes value. This property is subsequently based upon to produce persistent excitation at the entry of the linear subsystem.

Remark 1. (a) The mathematical definitions of the switch and backlash nonlinearities (i.e. Eqs. (2.3) and (2.4)) are introduced for completeness of the present treatment. In particular, (2.4) may look somewhat complex for not familiar readers. For this reason, the forthcoming developments are presented in a way that makes them comprehensible knowing only the intuitive behaviors of the switch and backlash operators as these were commented on previously.

(b) The somewhat complex appearance of Assumption 1 comes from the fact that the mathematical formulation must apply to complex cases i.e. nonlinearities with noninvertible and/or noncontinuous borders. Recall that this assumption only concerns the backlash case and is introduced to ensure (in that case) the identifiability of the nonlinearity borders. Specifically, situations like those illustrated by Fig. 5 must be discarded. The question is: how these situations can be recognized in practical applications? This is answered noticing that when $v(t)$ spans the interval $\left[\begin{array}{ll}v_{m} & v_{M}\end{array}\right]$ several times in both senses then, just after one cycle, the signal $u(t)$ becomes constant. Consequently, the working point $(v(t), u(t))$ moves along a horizontal segment located between by the points $\left(v_{m}, u_{M}\right)$ and $\left(v_{M}, u_{M}\right)$ in Fig. 5 . In the light of this observation it is seen that, in situations like that pointed out by Fig. 5, if $v(t)$ spans (several times in both senses) a not sufficiently wide interval $\left[\begin{array}{ll}v_{m} & v_{M}\end{array}\right]$ then the system output $y(t)$ becomes constant (up to noise effect) after a transient period.

(c) Except for Assumptions 1 and 2, the bordering functions $C_{a}\left(\right.$.) and $C_{d}($.$) can be arbitrary. In particular, they may be$ noninvertible and nonsmooth making possible to take into consideration rely-type nonlinearity (Fig. 6). Interestingly, the nonlinearity borders are even allowed to cross each other (Fig. 4). Therefore, the present work clearly represents a major progress compared to previous works (Bai, 2002; Cerone \& Regruto, 2007; Chaoui et al., 2005; Giri, Rochdi, Chaoui et al., 2008; Giri, Rochdi, Elayan et al., 2008).

\subsection{Identification objective and relevance of periodic exciting inputs}

The purpose is to determine, as accurately as possible, both the linear parametric subsystem model $B\left(q^{-1}\right) / A\left(q^{-1}\right)$ and the nonlinear dynamic operator $F[$.$] . Since the latter is nonpara-$ metric, the last objective amounts to determine a finite set of

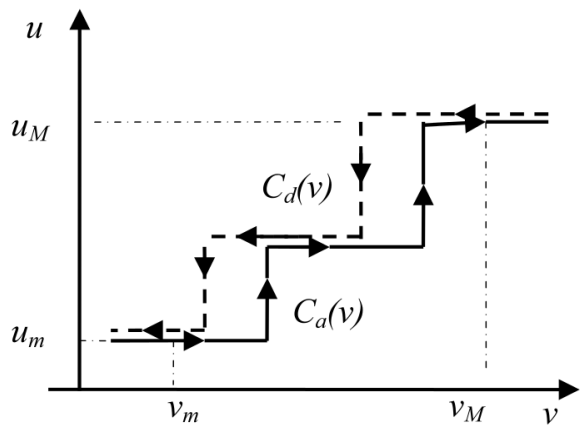

Fig. 6. Relay type operator.

points on each lateral borders. This is the usual practice when nonparametric nonlinearities are concerned. The points of interest are respectively denoted $\left\{\left(V_{j}^{d}, C_{d}\left(V_{j}^{d}\right)\right) ; j=1, \ldots, m\right\}$ and $\left\{\left(V_{j}^{a}, C_{a}\left(V_{j}^{a}\right)\right) ; j=1, \ldots, m\right\}$. The number $m$ is chosen by the user and may (in theory) be arbitrarily large. The abscissas $\left\{V_{1}^{d}, \ldots, V_{m}^{d}\right\}$ and $\left\{V_{1}^{a}, \ldots, V_{m}^{a}\right\}$ are arbitrarily selected by the user within the working interval $\left[v_{m}, v_{M}\right]$. A common difficulty to all Hammerstein systems lies in the inaccessibility of the inner sequence $u(t)$. System identification then must solely relies on the available data, namely the measurements of $v(t)$ and $y(t)$. A specific difficulty to the case of switch/backlash nonlinearities is that the working point $(v(t), u(t))$ can move along both nonlinearity borders and, in the backlash case, also along horizontal paths connecting these borders. Obviously, the shape of the trajectory $\left\{(v(t), u(t)), 0 \leq t \leq T_{0}\right\}$ (for a given $T_{0}>0$ ) differs from one experiment to an other, depending on the input signal. Presently, periodic signals are privileged not only for their simplicity but also because they generate steady-state limit cycles. Limit cycles that coincide with one (and only one) of the nonlinearity borders are presently preferred, because the operator $F[$.] can then be assimilated to a static nonlinearity. As a matter of fact, not any periodic input signal leads to such kind of limit cycle. To illustrate this point, consider the following simple triangular signal (of period $T$ ):

$v(t)= \begin{cases}V_{j}^{a} & \text { for } t=t_{k}+(j-1) T / 2 p ; j=1, \ldots, p \\ V_{j}^{d} & \text { for } t=t_{k}+(j-1) T .2 p ; j=p+1, \ldots, 2 p\end{cases}$

where $t_{k}=k T(k=0,1,2, \ldots)$ and $p$ is any positive integer such that $(T / 2 p)$ is in turn an integer. The $V_{j}^{a}$ 's and $V_{j}^{d}$ 's are such that $v_{m}<V_{1}^{a}<V_{2}^{a}<\cdots<V_{p}^{a}=v_{M}$ and $v_{M}>V_{p+1}^{d}>V_{p+2}^{d}>$ $\cdots>V_{2 p}^{d}=v_{m}$. This signal increases from $v_{m}$ to $v_{M}$ during the first half of each period and decreases from $v_{M}$ to $v_{m}$ during the rest of time. The working point $(v(t), u(t))$ then spans the limit cycle including the lateral branches, $\left\{\left(v, C_{a}(v)\right), v_{m} \leq v \leq v_{M}\right\}$ and $\left\{\left(v, C_{d}(v)\right), v_{M} \geq v \geq v_{m}\right\}$, and, in the backlash case only, the two (top and bottom) horizontal lines connecting these borders (Fig. 7). If the inner signal $u(t)$ were accessible to measurement, then the above experiment would make it possible to determine a finite number of points of the above lateral branches based on the measurements of $(v(t), u(t))$ and get an input-output representation (of the linear subsystem) involving linearly the unknown parameters $\left(a_{i}, b_{i}\right)$. Unfortunately, $u(t)$ is not accessible and is not necessarily persistently exciting (this in fact depends on the shape of the nonlinearity branches). This discussion emphasizes how much challenging the identification problem is when switch/backlash nonlinearities of arbitrary shape are involved. Then, it is not obvious to get limit cycles consisting of one (and only one) border. The present work aims at designing experiments such that the switch/backlash nonlinearity can actually be assimilated to a static nonlinearity and the involved excitation is persistent. 


\section{Descendent Identification Scheme (DIS)}

The DIS is conceived to determine the linear subsystem parameters $\left(a_{i}, b_{i}\right)$ as well as $m$ points located on the nonlinearity descendent border. The number $m$ and abscissas of points are arbitrarily chosen by the designer (within the interval $\left[v_{m}, v_{M}\right]$ ).

\subsection{Descendent Periodic (DP) experiment}

The DP-experiment involves a $T$-periodic input $v^{d}(t)$ such that, for all $t_{k}=k T(k=0,1,2, \ldots)$ and all $t \in\left\{t_{k}, \ldots, t_{k+1}-1\right\}$ :

$v^{d}(t)= \begin{cases}V_{j}^{d} & \text { for } t=t_{k}+\tau_{j} ; j=1, \ldots, m \\ v_{M} & \text { otherwise }\end{cases}$

where the real numbers $V_{j}^{d}$ are such that $v_{m}=V_{1}^{d}<V_{2}^{d}<\cdots<$ $V_{m}^{d}<v_{M}$ and the $m$ integers $\tau_{j}$ satisfy:

$\tau_{1} \geq 1, \quad \tau_{j}-\tau_{j-1} \geq 2(j=2, \ldots, m)$ and $\tau_{m}<T$.

The input signal $v^{d}(t)$ is a pulse type because it equals $v_{M}$ all time except at instants $t_{k}+\tau_{j}(j=1,2, \ldots, m)$. At these instants the input takes different values, namely $V_{j}^{d} \in\left\{V_{1}^{d}, V_{2}^{d}, \ldots, V_{m}^{d}\right\}$, which all are smaller than $v_{M}$. Fig. 11 illustrates the shape of this class of signals. In view of (2.3)-(2.4) and Assumption 1 (or simply inspecting Figs. 2a-2b), it follows from (3.1) that the working point $\left(v^{d}(t), u(t)\right)$ never gets to the 'ascending' branch $\left\{\left(v, C_{a}(v)\right), v_{m} \leq v \leq v_{M}\right\}$, except for the particular point $\left(v_{M}, u_{M}\right)$ (the only of that branch that is presently excited). Furthermore, note that the working point moves like in a 'satellitecommunication' process i.e. it never goes directly from one position $\left(V_{j}^{d}, C_{d}\left(V_{j}^{d}\right)\right)$ to the next one $\left(V_{j+1}^{d}, C_{d}\left(V_{j+1}^{d}\right)\right)$; it must transit by the 'satellite' position i.e. the point $\left(v_{M}, u_{M}\right)$ (Figs. $8 \mathrm{a}-8 \mathrm{~b}$ ). It is clearly seen that the working point $\left(v^{d}(t), u(t)\right)$ stays all time within a limit cycle, denoted $D_{l c}$ and referred to 'descendent limit cycle'. In the case of backlash nonlinearities, $D_{l c}$ is composed of the descendent branch $\left\{\left(v, C_{d}(v)\right), v_{m} \leq v \leq \max C_{d}^{-1}\left(u_{M}\right)\right\}$ and the top horizontal line connecting the above branch to the point $\left(v_{M}, u_{M}\right)$. In the switch case, $D_{l c}$ is composed of the single point $\left(v_{M}, u_{M}\right)$ and the descendent branch $\left\{\left(v, C_{d}(v)\right), v_{m} \leq v \leq \max C_{d}^{-1}\left(u_{M}\right)\right\}$ delimited by the vertical lines $v=v_{m}$ and $v=\max C_{d}^{-1}\left(u_{M}\right)=v_{M}$.

In both cases, the domain $D_{l c}$ is a simple curve inducing a standard function, denoted $F^{d}($.$) , such that D_{l c}=\left\{\left(v, F^{d}(v)\right)\right.$, $\left.v_{m} \leq v \leq v_{M}\right\}$. Specifically:

$F^{d}(v)= \begin{cases}C_{d}(v) & \text { if } v_{m} \leq v \leq \max C_{d}^{-1}\left(u_{M}\right) \\ u_{M} & \text { if } \max C_{d}^{-1}\left(u_{M}\right)<v \leq v_{M} .\end{cases}$

The data collected in the DP-experiment will next be used to estimate the points $\left(V_{j}^{d}, F^{d}\left(V_{j}^{d}\right)\right)(j=1,2, \ldots, m)$, as well as the linear subsystem parameters $\left(a_{i}, b_{i}\right)$.

Remark 2. It is seen (Fig. 8b) that, for switch nonlinearities, all $m$ points $\left(V_{j}^{d}, F^{d}\left(V_{j}^{d}\right)\right)$ are located on the descendent border. In the backlash case (Fig. 8a), some of the $m$ points $\left(V_{j}^{d}, F^{d}\left(V_{j}^{d}\right)\right)$, specifically those with higher abscissas, may be on the horizontal segment delimited by the points $\left(\max C_{d}^{-1}\left(u_{M}\right), u_{M}\right)$ and $\left(v_{M}, u_{M}\right)$. To enlarge the set of useful points (those belonging to the descendent branch), the abscissas $V_{j}^{d}$ should not be too close to $v_{M}$. The pulse and nonmonotonic nature of the sequence (3.1) will prove to be crucial in making this sequence persistently exciting (Theorem 3.1, parts 1 and 2). If the aim of the experiment were simply to keep the point $(v(t), u(t))$ moving on the descendent border, one could use simpler sequences.

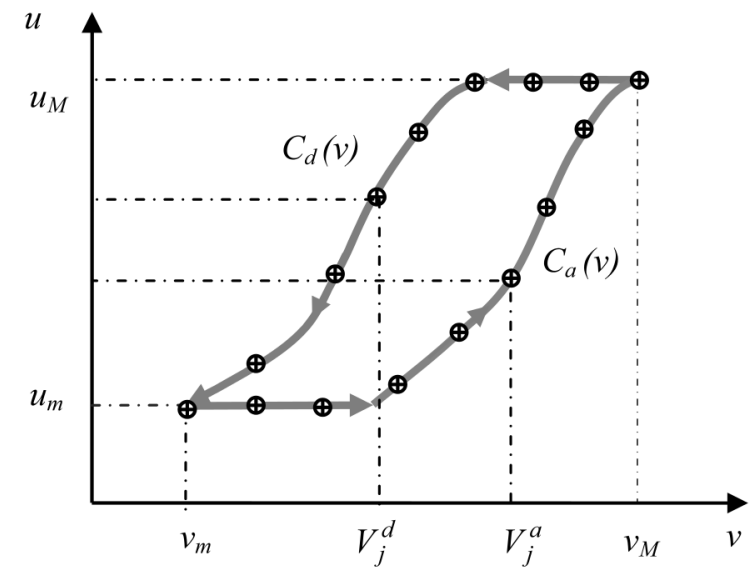

Fig. 7. Complete limit cycle obtained, with backlash operators, when $v(t)$ spans the interval $\left[v_{m} v_{M}\right]$ several times in both senses.

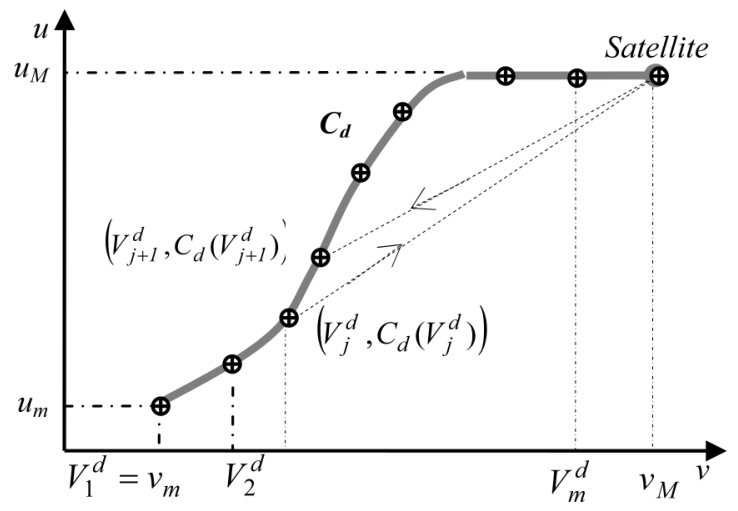

Fig. 8a. Movement of the working point $(v(t), u(t))$ on the limit cycle $D_{l c}$ during the DP-experiment-case of backlash nonlinearity.

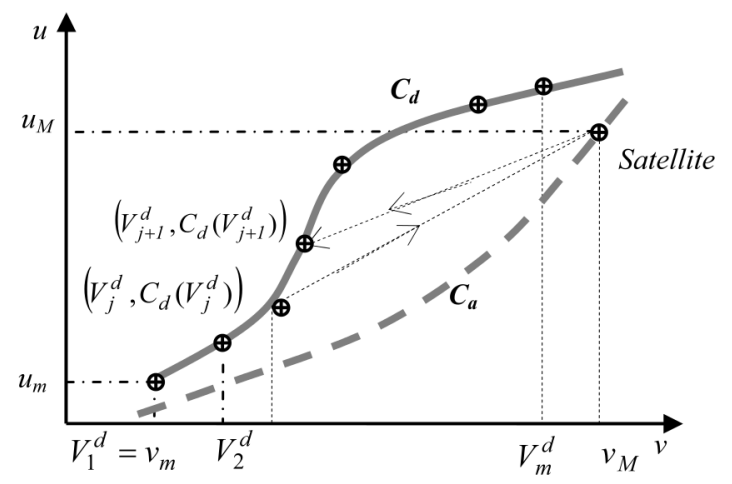

Fig. 8b. Movement of the working point $(v(t), u(t))$ on the limit cycle $D_{l c}$ during the DP experiment-case of switch nonlinearity.

\subsection{System parameterization}

DPE-model. It has already been mentioned that the trajectory of $\left(v^{d}(t), u(t)\right)$ stays all time on $D_{l c}$, occupying there the specific positions: $\left(v_{M}, u_{M}\right)$ and $\left(V_{j}^{d}, F^{d}\left(V_{j}^{d}\right)\right)(j=1, \ldots, m)$. Then, during the DP-experiment, the quantity $F^{d}\left(v^{d}(t)\right)$ may be substituted to $F\left[v^{d}\right](t)$ in the initial model (2.1) yielding a new model, referred to DPE-model:

$A\left(q^{-1}\right) y(t)=B\left(q^{-1}\right) F^{d}(v(t))+\xi(t) \quad$ with $v(t)=v^{d}(t)$

where $F^{d}($.$) is defined by (3.2). Note that the substitution of$ $F^{d}($.) to $F[$.] involves no error. Furthermore, the fact that the couple $\left(v^{d}(t), F^{d}\left(v^{d}(t)\right)\right)$ only occupies the positions $\left(v_{M}, u_{M}\right)$ 
and $\left(V_{j}^{d}, F^{d}\left(V_{j}^{d}\right)\right)(j=1, \ldots, m)$ will make it possible to fully parameterize the model (3.3). In order to achieve such full model parameterization, $F^{d}($.$) is first centered so that the obtained$ centered nonlinearity, denoted $\tilde{F}_{d}($.$) , satisfies \tilde{F}_{d}(0)=0$.

$D P E-$ model centering. Let $y_{0}^{d}(t)$ denote the response of $(2.1)$ to the following step input:

$v(t)=v_{0}^{d}(t) \stackrel{\text { def }}{=} \begin{cases}v_{m} & \text { if } t=0 \\ v_{M} & \text { if } t \geq 1 .\end{cases}$

It follows from (2.3)-(2.4) (or alternately from Figs. 8a-8b) that $u_{0}^{d}(t) \stackrel{\text { def }}{=} F\left[v_{0}^{d}\right](t)=u_{M}$, for all $t \geq 1$, whatever $u_{0}^{d}(0)$. As the point $\left(v_{M}, u_{M}\right)$ belongs to the domain $D_{l c}$, one also has $u_{0}^{d}(t)=$ $F^{d}\left(v_{0}^{d}(t)\right)=u_{M}$, for all $t \geq 1$. Therefore, the couple of signals $v_{0}^{d}(t)$ and $y_{0}^{d}(t)$ can be related by the general model (2.1) as well as by the DPE-model (3.3). Thus, one has for all $t \geq 1$ :

$A\left(q^{-1}\right) y_{0}^{d}(t)=B\left(q^{-1}\right) u_{0}^{d}(t)+\xi_{0}^{d}(t)$

where $\xi_{0}^{d}(t)$ denotes the realization of $\xi(t)$ during the present experiment. As $u_{0}^{d}(t)=u_{M}$ (for all $t \geq 1$ ) it follows time-averaging both sides of (3.5) (over the interval $1 \leq t \leq L$ ) that $^{2}$ :

$A(1) \bar{y}_{0}^{d}(L)=B(1) u_{M}+\bar{\xi}_{0}^{d}(L)$.

As $\xi_{0}^{d}(t)$ is zero mean and ergodic, one has $\bar{\xi}_{0}^{d}(L) \rightarrow 0$ as $L \rightarrow \infty$. Then, it follows from (3.6) that:

$A(1) \bar{y}_{0}^{d}=B(1) u_{M}$

where $\bar{y}_{0}^{d}$ denotes the limit of $\bar{y}_{0}^{d}(L)$ when $L \rightarrow \infty$. Practically $\bar{y}_{0}^{d}$ can be computed from a large data sample $\{y(t), t=1, \ldots, L\}$. Subtracting both sides of (3.7) from the corresponding sides in (3.3), gives:

for all $t \geq 0$

$A\left(q^{-1}\right)\left(y(t)-\bar{y}_{0}^{d}\right)=B\left(q^{-1}\right)\left(F^{d}(v(t))-u_{M}\right)+\xi(t)$.

For convenience, let us introduce the following notations:

$\tilde{v}(t) \stackrel{\text { def }}{=} v(t)-v_{M}, \quad \tilde{y}(t) \stackrel{\text { def }}{=} y(t)-\bar{y}_{0}^{d}$,

$\tilde{F}^{d}(w) \stackrel{\text { def }}{=} F^{d}\left(w+v_{M}\right)-u_{M}$

It is readily seen that:

$\tilde{F}^{d}(\tilde{v}(t)) \stackrel{\text { def }}{=} F^{d}(v(t))-u_{M}$.

Then, (3.8) can be given the following compact form, called centered DPE- model:

$A\left(q^{-1}\right) \tilde{y}(t)=B\left(q^{-1}\right) \tilde{F}^{d}(\tilde{v}(t))+\xi(t)$.

Note that the Hammerstein-like model (3.10) holds solely within the DP-experiment context (i.e. when $v=v^{d}$ ). It involves the static nonlinearity $\tilde{F}^{d}($.$) that is analytically defined in (3.9b). Further-$ more, using (3.2), (3.9a) and (3.9c), one readily gets:

$\tilde{F}^{d}(0)=0$

$\tilde{F}^{d}\left(W_{1}^{d}\right)=u_{m}-u_{M} \neq 0$

with $W_{1}^{d} \stackrel{\text { def }}{=} V_{1}^{d}-v_{M}=v_{m}-v_{M}$.

The fact that $u_{m}-u_{M} \neq 0$ is guaranteed by Assumption 2 . Properties (3.11a)-(3.11b) will prove to be crucial in achieving a

\footnotetext{
2 Throughout the paper, $\bar{x}(L)$ denotes the arithmetic average of a sequence $x(t)$ over the time interval $[0 L]$ i.e. $\bar{x}(L)=\frac{1}{L} \sum_{t=0}^{L-1} x(t)$. In the case of stationary ergodic random sequences, one has $E(x(t))=\lim _{L \rightarrow \infty} \bar{x}(L)$, for all $t$.
}

persistent excitation property (Theorem 3.1). For now, recall that the Hammerstein model (3.10) is not uniquely defined. Indeed, if the triplet $\left(A\left(q^{-1}\right), B\left(q^{-1}\right), \tilde{F}^{d}[].\right)$ is representative of the system, then so is any triplet of the form $\left(A\left(q^{-1}\right), K B\left(q^{-1}\right), \tilde{F}^{d}[] / K.\right)$ with $K \neq 0$.

To get off of such model multiplicity we will, from now on, focus on the unique model that satisfies ${ }^{3}$ :

$\tilde{F}^{d}\left(W_{1}^{d}\right)=-1$.

The existence and uniqueness of such model is guaranteed by (3.11b). Recall that the initial identification purpose is to determine the coefficients of the polynomial pair $\left(A\left(q^{-1}\right), B\left(q^{-1}\right)\right)$ and the $m$ points $\left(V_{j}^{d}, F^{d}\left(V_{j}^{d}\right)\right)(j=1,2, \ldots, m)$ of the $F^{d}$-graphical characteristic. It follows from (3.9b) that the last requirement amounts to determining the following $m$ points of $\tilde{F}^{d}$ :

$\left(W_{j}^{d}, \tilde{F}^{d}\left(W_{j}^{d}\right)\right) ; \quad j=1,2, \ldots, m$

with $W_{j}^{d} \stackrel{\text { def }}{=} V_{j}^{d}-v_{M}$

To this end, we let $v(t)=v^{d}(t)$ so that $\tilde{v}(t)=\tilde{v}^{d}(t) \stackrel{\text { def }}{=}$ $v^{d}(t)-v_{M}$ is in turn a pulse-type periodic signal with the same period as $v^{d}(t)$. Specifically, one obtains from (3.1) and (3.12) that, for all $t_{k}=k T(k=0,1,2, \ldots)$ and all $t_{k} \leq t<t_{k+1}$ :

$\tilde{v}^{d}(t)= \begin{cases}W_{j}^{d} & \text { for } t=t_{k}+\tau_{j}, j=1,2, \ldots, m \\ 0 & \text { otherwise }\end{cases}$

where the $\tau_{j}$ 's are as in (3.1). Clearly, $\tilde{v}^{d}(t)$ takes values in the set $\left\{0, W_{j}^{d} ; j=1,2, \ldots, m\right\}$ and, consequently, the trajectory $\left\{\left(\tilde{v}^{d}(t), \tilde{F}^{d}\left(\tilde{v}^{d}(t)\right)\right) t \geq 0\right\}$ consists of $m+1$ points: the $m$ points of the set in (3.12) and the origin $(0,0)$. Therefore, the function $\tilde{F}^{d}\left(v^{d}(t)\right)$ can be given (within the present experiment context) an exact representation with a $m$ th-degree polynomial.

Polynomial representation of $\tilde{F}^{d}\left(\tilde{v}^{d}(t)\right)$. Let $P^{d}(w)$ denotes the unique $m$ th-degree polynomial interpolating the set of points including the origin $(0,0)$ and $\left(W_{j}^{d}, \tilde{F}^{d}\left(W_{j}^{d}\right)\right) ;(j=1,2, \ldots, m)$. Such polynomial is described using the Vandermonde formula:

$P^{d}(w)=w \sum_{j=1}^{m} c_{j}^{d} P_{j}^{d}(w)$

$c_{j}^{d}=\frac{\tilde{F}^{d}\left(W_{j}^{d}\right)}{W_{j}^{d}} ; \quad P_{j}^{d}(w) \stackrel{\text { def }}{=} \prod_{\substack{i=1 \\ i \neq j}}^{m} \frac{w-W_{i}^{d}}{W_{j}^{d}-W_{i}^{d}}$

It is easily seen that:

$P_{j}^{d}\left(W_{i}^{d}\right)=\delta_{i j} \stackrel{\text { def }}{=} \begin{cases}1 & \text { if } i=j \\ 0 & \text { if } i \neq j\end{cases}$

$P^{d}(0)=0 ; \quad P^{d}\left(W_{j}^{d}\right)=\tilde{F}^{d}\left(W_{j}^{d}\right) ; \quad j=1, \ldots, m$.

Presently, $\tilde{v}^{d}(t)$ only takes values in the set $\left\{0, W_{j}^{d} ; j=1,2\right.$, $\ldots, m\}$. Then, we have $\tilde{F}^{d}\left(\tilde{v}^{d}(t)\right)=P^{d}\left(\tilde{v}^{d}(t)\right)$, for all $t$. Therefore, $P^{d}\left(\tilde{v}^{d}(t)\right)$ can be substituted to $\tilde{F}^{d}\left(\tilde{v}^{d}(t)\right)$ in the DPE-centered model (3.10), implying for all $t \geq 0$ :

$A\left(q^{-1}\right) \tilde{y}(t)=B\left(q^{-1}\right) P^{d}\left(\tilde{v}^{d}(t)\right)+\xi(t)$.

\footnotetext{
3 If (3.11c) is not satisfied then (3.11a)-(3.11b) suggests that we focus on the unique model $\left(A\left(q^{-1}\right), K B\left(q^{-1}\right), \tilde{F}^{d} / K\right)$ with $K=-\left(u_{m}-u_{M}\right)$.
} 
The main advantage of (3.17) (over (3.10)) lies in the parametric feature of its nonlinearity (i.e. $\left.P^{d}().\right)$ making possible the achievement of regression forms. It is worthy pointing out that the passage from (3.10) to (3.17) entails no error, as long as the system is excited by the input sequence $v^{d}(t)$, generated according to (3.1).

DPE regression form. Using (3.14a), one obtains from (3.17):

$A\left(q^{-1}\right) \tilde{y}(t)=\sum_{i=1}^{n} \sum_{j=1}^{m} \mu_{i j}^{d} \tilde{v}^{d}(t-i) P_{j}^{d}\left(\tilde{v}^{d}(t-i)\right)+\xi(t)$

with $\mu_{i j}^{d}=b_{i} c_{j}^{d}$. Eq. (3.18) is given the following linear regression form:

$$
\begin{aligned}
& \tilde{y}(t)=\Phi^{d}(t)^{T} \Theta^{d}+\xi(t) \\
& \Phi^{d}(t)^{T}=[-\tilde{y}(t-1) \ldots-\tilde{y}(t-n) \\
& \tilde{v}^{d}(t-1) P_{1}^{d}\left(\tilde{v}^{d}(t-1)\right) \ldots \tilde{v}^{d}(t-n) P_{1}^{d}\left(\tilde{v}^{d}(t-n)\right) \\
& \text {..... } \\
& \left.\tilde{v}^{d}(t-1) P_{m}^{d}\left(\tilde{v}^{d}(t-1)\right) \ldots \tilde{v}^{d}(t-n) P_{m}^{d}\left(\tilde{v}^{d}(t-n)\right)\right] \\
& \Theta^{d}=\left[\begin{array}{lllllllllll}
a_{1} & \ldots & a_{n} & \mu_{11}^{d} & \ldots & \mu_{n 1}^{d} & \ldots & \ldots & \mu_{1 m}^{d} & \ldots & \mu_{n m}^{d}
\end{array}\right]^{T} \text {. }
\end{aligned}
$$

As $\Theta^{d}$ comes in linearly, Eq. (3.19a) turns out to be an adequate parameterization to get estimates of the parameters $a_{i}$ and $\mu_{i j}^{d}$. But, the question is how to recover estimates of $\left(b_{i}, c_{j}^{d}\right)$ from those of $\mu_{i j}^{d}$ ? A subsidiary question is how to recover $F^{d}\left(V_{j}^{d}\right)$ from the previous estimates? These questions are now answered making use of the available information. First, one gets from (3.14b) and (3.11c):

$c_{1}^{d}=\frac{\tilde{F}^{d}\left(W_{1}^{d}\right)}{W_{1}^{d}}=-\frac{1}{W_{1}^{d}}$.

That is, the coefficient $c_{1}^{d}$ is perfectly known (because so is $W_{1}^{d}$ ). Then, using (3.18) one obtains:

$b_{i}=\frac{\mu_{i 1}^{d}}{c_{1}^{d}}=-\mu_{i 1}^{d} W_{1}^{d} \quad(i=1, \ldots, n)$.

The $c_{j}^{d}$ s $s$ are in turn obtained multiplying the second equation in (3.18) by $b_{i}$ and summing the resulting equalities over $i=$ $1, \ldots, n$. Doing so, one gets:

$c_{j}^{d}=\frac{\sum_{i=1}^{n} b_{i} \mu_{i j}^{d}}{\sum_{i=1}^{n} b_{i}^{2}} \quad(j=2, \ldots, m)$.

Finally, (3.14b)-(3.16) yields $\tilde{F}^{d}\left(W_{j}^{d}\right)=c_{j}^{d} W_{j}^{d}$ which, together with (3.9b), (3.12) and (3.7), implies:

$F^{d}\left(V_{j}^{d}\right)=c_{j}^{d} W_{j}^{d}+\frac{A(1)}{B(1)} \bar{y}_{0}^{d}, \quad(j=1, \ldots, m)$.

\subsection{Parameter estimation}

Based on the regression (3.19a)-(3.19c), the vector $\Theta^{d}$ can be estimated using the least squares estimator:

$\hat{\Theta}^{d}(N)=\left[\frac{1}{N} \sum_{i=1}^{N} \Phi^{d}(i) \Phi^{d}(i)^{T}\right]^{-1}\left[\frac{1}{N} \sum_{i=1}^{N} \Phi^{d}(i) \tilde{y}(i)^{T}\right]$.

Estimates $\hat{a}_{i}(N), \hat{\mu}_{i j}(N)$ of $a_{i}$ and $\mu_{i j}$ are immediately obtained from $\hat{\Theta}^{d}(N)$, using (3.19c). Then, relations (3.20b)-(3.20d) suggest the following estimators for $b_{i}, c_{j}$ and $F^{d}\left(V_{j}^{d}\right)(j=2, \ldots, m ; i=$ $1, \ldots, n)$ :

$\hat{b}_{i}(N)=-\hat{\mu}_{i 1}(N) W_{1}^{d} \quad(i=1, \ldots, n)$

$\hat{c}_{j}^{d}(N)=\frac{\sum_{i=1}^{n} \hat{b}_{i}(N) \hat{\mu}_{i j}(N)}{\sum_{i=1}^{n} \hat{b}_{i}^{2}(N)} \quad(j=2, \ldots, m)$

$\hat{F}^{d}\left(V_{j}^{d}, N\right)=\hat{c}_{j}^{d}(N) W_{j}^{d}+\frac{\hat{A}(1, N)}{\hat{B}(1, N)} \bar{y}_{0}^{d}(N)$.

To show that all estimators are consistent, let us consider the undisturbed version of model (3.17), i.e.

$A\left(q^{-1}\right) z^{d}(t)=B\left(q^{-1}\right) P^{d}\left(\tilde{v}^{d}(t)\right)$

Introduce the following undisturbed version of the regression vector $\Phi^{d}(t)$ :

$$
\begin{aligned}
& Z^{d}(t)^{T}=\left[-z^{d}(t-1) \ldots-z^{d}(t-n)\right. \\
& \quad \tilde{v}^{d}(t-1) P_{1}^{d}\left(\tilde{v}^{d}(t-1)\right) \ldots \tilde{v}^{d}(t-n) P_{1}^{d}\left(\tilde{v}^{d}(t-n)\right) \\
& \quad \ldots \ldots \\
& \left.\quad \tilde{v}^{d}(t-1) P_{m}^{d}\left(\tilde{v}^{d}(t-1)\right) \ldots \tilde{v}^{d}(t-n) P_{m}^{d}\left(\tilde{v}^{d}(t-n)\right)\right] .
\end{aligned}
$$

With these notations, the main consistency results are formulated as follows:

Theorem 3.1. Consider the system (2.1), subject to Assumptions 1 and 2 , and excited by the periodic signal $v^{d}(t)$ defined by (3.1). Let the period $T$ and the numbers $\tau_{j}$ in (3.1) be chosen as follows:

$T=n(m+3) ; \quad \tau_{1}=2 n ;$
$\tau_{j}=(2+j) n \quad(j=2, \ldots, m)$.

(1) The state vector $Z^{d}(t)$, defined by (3.24a)-(3.24b), has the following strong persistent excitation property:

$\sum_{i=0}^{T} Z^{d}\left(t_{k}+i\right) Z^{d}\left(t_{k}+i\right)^{T} \geq \lambda I ; \quad$ for all $k$

where I denotes the identity matrix (with appropriate dimension) and $\lambda>0$ is a real constant.

(2) The state vector $\Phi^{d}(t)$ defined by (3.19b) possesses the following weak persistent excitation property:

$\lim _{N \rightarrow \infty} \frac{1}{N} \sum_{i=1}^{N} \Phi^{d}(t) \Phi^{d}(t)^{T}>\beta I, \quad$ w.p.1,

for some real constant $\beta>0$.

(3) The estimators (3.21) to (3.23) are consistent i.e. $\hat{\Theta}^{d}(N), \hat{b}_{i}(N)$, $\hat{c}_{j}^{d}(N)$ and $\hat{F}^{d}\left(V_{j}^{d}, N\right)(i=1, \ldots, n, j=1, \ldots, m)$ all converge (w.p.1) to their true values.

Proof of Part 1. This part is the key step towards the aimed consistency results. The main difficulty to prove it lies in the fact that the regressor $\Phi^{d}(t)$ in (3.19a) depends nonlinearly on the input sequence $\tilde{v}^{d}(t)$ i.e. (3.19a) is not a linear regression though $\Phi^{d}(t)$ comes in linearly. The same remarks apply to $Z^{d}(t)$ in $(3.24 \mathrm{a})-(3.24 \mathrm{~b})$. The detailed proof that $Z^{d}(t)$ is strongly persistently exciting (Part 1 ) is a bit long and technical. To alleviate the paper presentation, that proof is placed in the Appendix. Note simply that it relies on a technical lemma in Giri, Chaoui, and Rochdi (2002) where interval excitation by impulse sequences is analyzed. 
Proof of Part 2. Comparing, on one hand, (3.17) and (3.24a) and, on the other hand, (3.19b) and (3.24b), it follows that:

$$
\begin{aligned}
\Phi^{d}(t) & =Z^{d}(t)+\frac{1}{A\left(q^{-1}\right)}[\xi(t-1) \ldots \xi(t-n) 0 \ldots 0]^{T} \\
& \stackrel{\text { def }}{=} Z^{d}(t)+\chi(t-1) .
\end{aligned}
$$

Squaring both sides of (3.26) and summing the obtained equalities over $t=1, \ldots, N$, gives:

$$
\begin{aligned}
\frac{1}{N} \sum_{t=1}^{N} \Phi^{d}(t) \Phi^{d}(t)^{T} \\
=\frac{1}{N} \sum_{t=1}^{N} Z^{d}(t) Z^{d}(t)^{T}+\frac{1}{N} \sum_{t=1}^{N} \chi(t-1) \chi(t-1)^{T} \\
\quad+\frac{1}{N} \sum_{t=1}^{N} Z^{d}(t) \chi(t-1)^{T}+\frac{1}{N} \sum_{t=1}^{N} \chi(t-1) Z^{d}(t)^{T} .
\end{aligned}
$$

As the sequence $\{\xi(t)\}$ is ergodic and uncorrelated with $z^{d}(t)$, the last two terms on the right side of (3.27) converge in probability to zero as $N \rightarrow \infty$. Then, one gets:

$$
\begin{aligned}
\lim _{N \rightarrow \infty} \frac{1}{N} \sum_{t=1}^{N} \Phi^{d}(t) \Phi^{d}(t)^{T}= & \lim _{N \rightarrow \infty} \frac{1}{N} \sum_{t=1}^{N} Z^{d}(t) Z^{d}(t)^{T} \\
& +\lim _{N \rightarrow \infty} \frac{1}{N} \sum_{t=1}^{N} \chi(t-1) \chi(t-1)^{T} \\
\geq & \lim _{N \rightarrow \infty} \frac{1}{N} \sum_{t=1}^{N} Z^{d}(t) Z^{d}(t)^{T} .
\end{aligned}
$$

This, together with Part 1, proves Part 2 using the fact that $t_{k+1}-$ $t_{k}=T(\forall k)$.

Proof of Part 3. It is well known that the least squares estimator (3.21) is consistent in the presence of the persistent excitation property of Part 2. Furthermore, comparing (3.22a) and (3.20b) yields $\hat{b}_{i}(N)-b_{i}=\left(\mu_{i 1}-\hat{\mu}_{i 1}(N)\right) W_{1}^{d}$. The consistency of (3.22a) is a direct consequence of the consistency of $\hat{\Theta}^{d}(N)$ (as this particularly ensures that $\mu_{i 1}-\hat{\mu}_{i 1}(N)$ converges in probability to zero as $N \rightarrow \infty)$. The consistency of (3.22b) is proved similarly using the fact that both $\hat{\mu}_{i 1}(N)$ and $\hat{b}_{i}(N)$ are consistent estimators. Finally, comparing (3.23) and (3.20d) yields:

$$
\begin{aligned}
& \hat{F}_{j}^{d}\left(V_{j}^{d}, N\right)-F^{d}\left(V_{j}^{d}\right) \\
& \quad=W_{j}^{d}\left(\hat{c}_{j}^{d}(N)-c_{j}^{d}\right)+\frac{\hat{A}(1, N)}{\hat{B}(1, N)} \bar{y}_{0}^{d}(N)-\frac{A(1)}{B(1)} \bar{y}_{0}^{d} .
\end{aligned}
$$

The consistency of (3.23) then follows from the consistency of $\hat{c}_{j}^{d}(N), \hat{a}_{i}(N), \hat{b}_{i}(N)$ and $\bar{y}_{0}^{d}(N)$

\subsection{DIS practical considerations}

Practical procedure. The application of the DIS is performed following the next steps:

1. Working interval choice. The interval $\left[\begin{array}{ll}v_{m} & v_{M}\end{array}\right]$ is arbitrary in the presence of switch operators. In the backlash case, [ $\left.v_{m} v_{M}\right]$ must be sufficiently large to allow the identifiability of the nonlinearity borders. To this end, proceed as follows:

(a) apply any signal oscillating between $v_{m}$ and $v_{M}$ and observe the system output $y(t)$;

(b) if $y(t)$ is constant (up to noise) in steady state then enlarge the interval $\left[\begin{array}{ll}v_{m} & v_{M}\end{array}\right]$ and go to $(\mathrm{a})$; (c) else, keep the current interval and select therein the abscissas $V_{j}^{d}(j=1, \ldots, m)$ of the points to be determined on the descendent border, respecting the condition $v_{m}=$ $V_{1}^{d}<V_{2}^{d}<\cdots<V_{m}^{d}<v_{M}$.

2. Apply the step input signal $v(t)=v_{0}^{d}(t)$ defined by (3.4) and compute the mean value $\bar{y}_{0}^{d}$ of the resulting system response, over a sufficiently large observation interval.

3. Apply the sequence $v^{d}(t)$ defined by (3.1) taking into account Theorem 3.1 regarding the choice of the $\tau_{j}$ 's. Get a sample of the resulting system response $y(t)(t=1, \ldots, N$, for a sufficiently large $N$ ).

4. Construct the centered signals defined by (3.9a) (i.e. $\tilde{v}(t)=$ $v(t)-v_{M}$ and $\left.\tilde{y}(t)=y(t)-\bar{y}_{0}^{d}\right)$ for $t=1, \ldots, N$. Construct the vector sequence $\Phi^{d}(t)$ defined by (3.19b).

5. Compute the estimates $\hat{\Theta}^{d}(N), \hat{b}_{i}(N), \hat{c}_{j}^{d}(N)$ and $\hat{F}^{d}\left(V_{j}^{d}, N\right)(i=$ $1, \ldots n, j=1, \ldots, m)$ using the estimators (3.21) to (3.23).

6. End of the identification procedure.

For numerical computations reason, the number $m$ should not be too large. If a large set of points is desired, it would be preferable to repeat the above identification procedure several times focusing each time on a different subset of points.

On polynomial interpolation of identified points

The DIS design involves the substitution of $P^{d}\left(\tilde{v}^{d}(t)\right)$ to $\tilde{F}^{d}\left(\tilde{v}^{d}(t)\right)$ in the DPE-centered model (3.10). It must be emphasized that this substitution, introduced to parameterize the considered identification problem, entails no error as long as the model (3.10) is excited by a sequence that takes its values in the set $\left\{0, W_{j}^{d} ; j=1,2, \ldots, m\right\}$, which is precisely the case of the sequence $\tilde{v}^{d}(t)$ due to (3.13). However, one must not conclude that the polynomial $\hat{P}^{d}(w)=w \sum_{j=1}^{m} \hat{c}_{j}^{d} P_{j}^{d}(w)$, induced by the estimates $\hat{c}_{j}^{d}$, approximates well the nonlinear function $\tilde{F}^{d}$ over the whole interval $\left[\begin{array}{ll}v_{m} & v_{M}\end{array}\right]$. The identification scheme aim was not the determination of the whole descendent border but just $m$ points on it. This goal has actually been achieved since the couples $\left(W_{j}^{d}, \hat{P}^{d}\left(W_{j}^{d}\right)\right)$ are shown to be consistent estimates of the points of interest i.e. $\left(W_{j}^{d}, \tilde{F}^{d}\left(W_{j}^{d}\right)\right) ;(j=1,2, \ldots, m)$. Now, if one seeks a polynomial approximation of $\tilde{F}^{d}$, it must be resorted to suitable methods involving e.g. spline interpolation using the obtained estimates $\left(W_{j}^{d}, \hat{P}^{d}\left(W_{j}^{d}\right)\right)$, see e.g. Phillips (2003).

\subsection{DIS simulation}

The proposed identification approach is now illustrated considering a Hammerstein-like system with a backlash nonlinearity $F[$.] (that may represent e.g. an actuator). The linear subsystem is initially characterized (before system rescaling) by:

$A_{\text {init }}\left(q^{-1}\right)=1+q^{-1}+0.21 q^{-2}$;

$B_{\text {init }}\left(q^{-1}\right)=q^{-1}+0.5 q^{-2}$.

The initial operator $F[$.$] is bordered by the functions:$

$C_{\text {init }}^{d}(v)=v^{3}+3.5 v^{2}+2.75 v+4.625 ;$

$C_{\text {init }}^{a}(v)=C_{\text {init }}^{d}(v-1)$.

The error $\xi(t)$ in (2.1) is presently a band-limited white-noise with variance 16 . The working interval is $\left[\begin{array}{ll}v_{m} & v_{M}\end{array}\right]=\left[\begin{array}{ll}-3 & 4\end{array}\right]$ and the number of points to be determined on the descendent border is $m=6$ and their abscissas are:

$v_{m}=V_{1}^{d}=-3 ; \quad V_{2}^{d}=-2 ; \quad V_{3}^{d}=-1 ;$

$V_{4}^{d}=1 ; \quad V_{5}^{d}=2 ; \quad V_{6}^{d}=3$. 
One readily gets $u_{m}=C_{\text {init }}^{d}\left(v_{m}\right)=0.875$ and $u_{M}=C_{\text {init }}^{a}\left(v_{M}\right)=$ 71.375. The initial model, (3.28)-(3.29), is now rescaled so that property (3.11c) holds. Accordingly, the polynomial $B_{\text {init }}\left(q^{-1}\right)$ is multiplied by $K=u_{M}-u_{m}$ and $F[$.] is divided by the same scaling factor. Doing so, one gets a new model which is also representative of the system. It is readily checked that $K=u_{M}-u_{m}=70.5$. The new linear subsystem model turns out to be characterized by the polynomial operators:

$A\left(q^{-1}\right)=A_{\text {init }}\left(q^{-1}\right)=1+q^{-1}+0.21 q^{-2}$;

$B\left(q^{-1}\right)=K B_{\text {init }}\left(q^{-1}\right)=70.5 q^{-1}+35.25 q^{-2}$.

The new nonlinearity is characterized by the following borders (see Fig. 9):

$C^{d}(v)=\frac{C_{\text {init }}^{d}(v)}{K}$ and $C^{a}(v)=\frac{C_{i n i t}^{a}(v)}{K}$.

\subsubsection{DIS application}

Following the identification procedure in Section 3.4, the step signal (3.4) is first applied. From the resulting step response we obtain the estimate $\hat{\bar{y}}_{0}^{d}=16.15$ of $\bar{y}_{0}^{d}$, with $L=2000$ in (3.7). Then, the signal $v^{d}(t)$ defined by (3.1), and characterized by (3.25) and (3.30), is applied and the obtained system response is plotted in Fig. 11. We then proceed with data centering according to (3.9a). The obtained centered data are used to construct the regressor sequence $\Phi^{d}(t)(t=1, \ldots, 2000)$. Using this sequence in the estimator (3.21) one gets the following estimate:

$$
\begin{aligned}
\hat{\Theta}^{d}= & {\left[\begin{array}{llllll}
1.0005 & 0.2122 & 10.0769 & -5.0439 & 11.1040 \\
& -5.5432 & 13.4025 & -6.7368 & 19.8480 & -10.027 \\
& 19.8956 & -10.0071 & 0.6476 & -0.0617
\end{array}\right] . }
\end{aligned}
$$

This is based upon to get the remaining estimates using the estimators to (3.22)-(3.23). The obtained estimates are shown, together with their true values, in Tables 1 and 2. Finally, the estimated points on the descendent border are shown by Fig. 10. Such good estimation quality is further illustrated by Fig. 11 which shows the responses of the true system and the estimated model when the signal $v^{d}(t)$ is applied to both.

\subsubsection{DIS consistency checking}

To check the consistency of the estimator (3.21), the above experiment has been repeated 1000 times with different realizations of the noise $\xi(t)$. The estimate of $\Theta^{d}$ obtained at the $i$ th experiment is denoted $\hat{\Theta}^{d, i}(i=1 \cdots 1000)$. Fig. 12 shows the distribution of the third component of $\hat{\Theta}^{d, i}$ which represents the estimate of $\mu_{1,1}^{d}=b_{1} c_{1}^{d}$ (see (3.19)). It is seen that the estimates remain in the vicinity of the true parameter value, equal to 10.07 (see (3.31)). On the other hand, let $P_{\varepsilon}$ denotes the probability that $\left\|\hat{\Theta}^{d}-\Theta^{d}\right\| /\left\|\Theta^{d}\right\|<\varepsilon$ and $P_{\varepsilon, L}$ the estimate of $P_{\varepsilon}$ obtained using the results of the first $L$ experiments (i.e. DIS repetitions). From the above 1000 experiments, we got the following values:

$P_{\varepsilon, L}= \begin{cases}0.97 & \text { with } \varepsilon=0.02 \text { and } L=100 \\ 0.995 & \text { with } \varepsilon=0.02 \text { and } L=1000\end{cases}$

This confirms the consistency of the estimator (3.21), despite the not negligible noise (variance 16 ).

\subsubsection{DIS robustness checking}

To complete the identification scheme evaluation, let us check its robustness to modeling error. Specifically, suppose that the true system is rigorously described by the following model:

$A\left(q^{-1}\right) y(t)=B\left(q^{-1}\right) u(t)+\xi(t)$

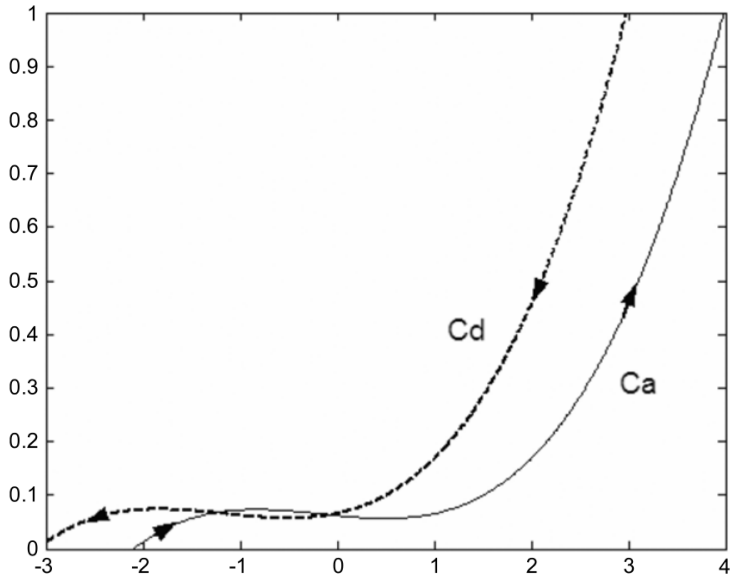

Fig. 9. Lateral borders of the considered backlash operator (simulation of Section 3.5).

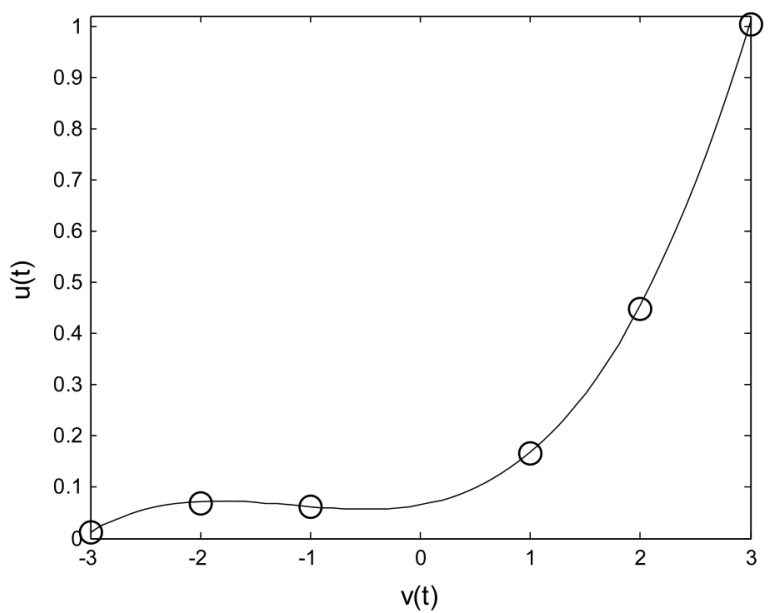

Fig. 10. Descendent nonlinearity border; the circles indicate the estimated points $\left(V_{j}^{d}, \hat{F}^{d}\left(V_{j}^{d}\right)\right)$.

Table 1

Parameters of the linear subsystem characterized by (3.31) and their estimates.

\begin{tabular}{lllll}
\hline$j$ & $a_{j}$ & $\hat{a}_{j}$ & $b_{j}$ & $\hat{b}_{j}$ \\
\hline 1 & 1 & 1.001 & 70.5 & 70.48 \\
2 & 0.21 & 0.2111 & 35.25 & 35.27 \\
\hline
\end{tabular}

Table 2

Parameters of the nonlinearity characterized by (3.32) and their estimates.

\begin{tabular}{lllllll}
\hline$V_{j}^{d}$ & -3 & -2 & -1 & 1 & 2 & 3 \\
\hline$F^{d}\left(V_{j}^{d}\right)$ & 0.0124 & 0.073 & 0.062 & 0.168 & 0.456 & 1.01 \\
$\hat{F}^{d}\left(V_{j}^{d}\right)$ & 0.0122 & 0.068 & 0.061 & 0.167 & 0.447 & 1.00 \\
\hline
\end{tabular}

with $u=F[w]$ and $w(t)=G\left(q^{-1}\right) v(t)$. Compared to the supposed model, i.e. (2.1), (3.34) involves a first order dynamics, preceding the backlash operator $F\left[\right.$.]. Typically, $G\left(q^{-1}\right)=(1-\lambda) /\left(1-\lambda q^{-1}\right)$ with small $0<\lambda<1$. Obviously, the presence of the dynamics of $G\left(q^{-1}\right)$ is totally ignored in the identification scheme DIS which is applied exactly as in Section 3.5.1. In particular, the input signal and system reforming characterized by (3.30)-(3.32) are kept unchanged. The only change is that the output $y(t)$ is presently influenced by the neglected dynamics $G\left(q^{-1}\right)$. Table 3 shows the estimates obtained by the DIS scheme for different values of $0<$ $\lambda<1$. The estimates obtained when $\lambda \neq 0$ deviate slightly from those of the case $\lambda=0$. This proves the robustness of the DIS scheme to input modeling errors. 
Table 3

Parameter estimates in the presence of the dynamics $G\left(q^{-1}\right)$.

\begin{tabular}{|c|c|c|c|c|c|c|c|c|c|}
\hline & $a_{1}$ & $a_{2}$ & $b_{1}$ & $b_{2}$ & $\tilde{F}^{d}\left(V_{2}^{d}\right)$ & $\tilde{F}^{d}\left(V_{3}^{d}\right)$ & $\tilde{F}^{d}\left(V_{4}^{d}\right)$ & $\tilde{F}^{d}\left(V_{5}^{d}\right)$ & $\tilde{F}^{d}\left(V_{6}^{d}\right)$ \\
\hline True values & 1 & 0.21 & 70.5 & -35.3 & -0.939 & -0.950 & -0.844 & -0.557 & 0 \\
\hline Estimates when $\lambda=0$ & 0.996 & 0.21 & 70.5 & -35.4 & -0.938 & -0.9560 & -0.844 & -0.552 & 0.002 \\
\hline Estimates when $\lambda=0.005$ & 0.968 & 0.19 & 70.9 & -34.0 & -0.9343 & -0.9564 & -0.854 & -0.565 & -0.012 \\
\hline Estimates when $\lambda=0.01$ & 1.04 & 0.24 & 67.7 & -36.48 & -0.9306 & -0.955 & -0.861 & -0.575 & -0.026 \\
\hline
\end{tabular}
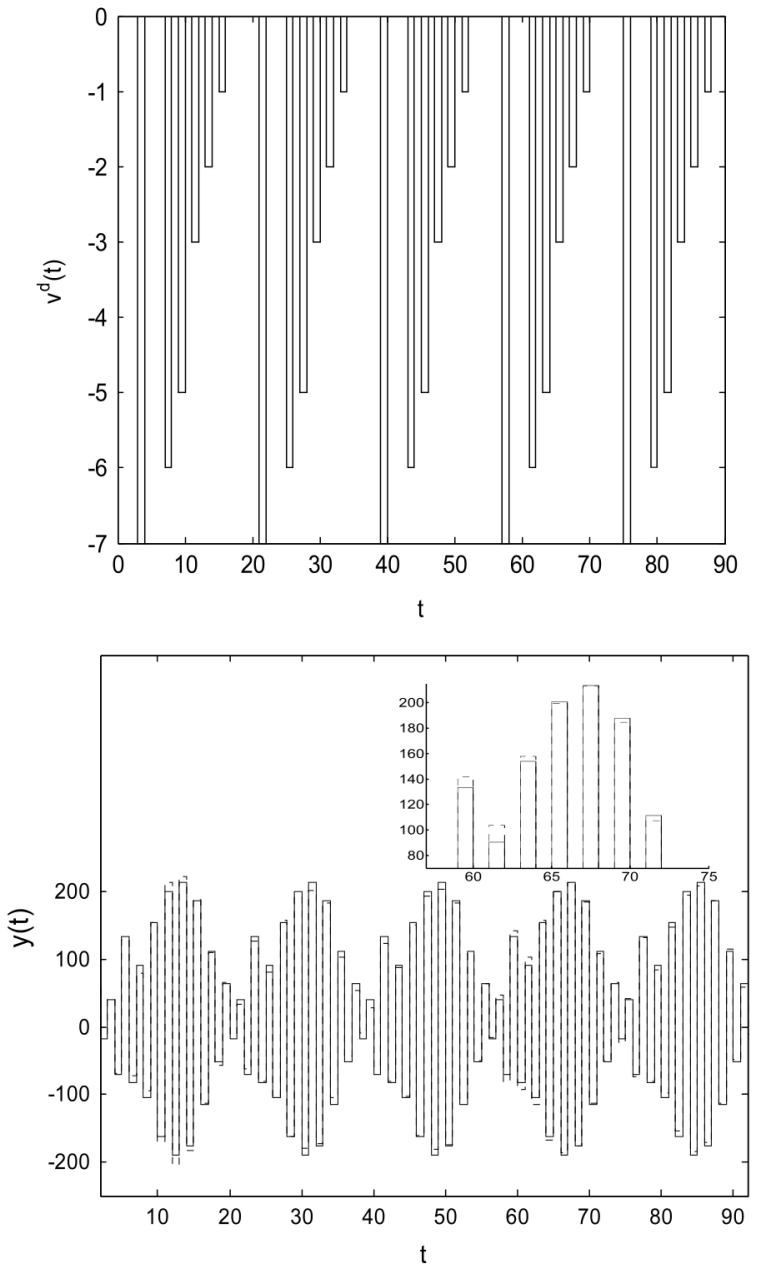

Fig. 11. Signals generated in the DP-experiment (over two periods). Above: input signal $v^{d}(t)$ generated by (3.1). Below: system outputs $y(t)$ (solid) and model output induced by $\hat{\Theta}^{d}(N)$ (dashed). For the sake of clarity, the plotted signals are in fact hold-versions of the discrete-time signals and a zoom is made on the time interval [55 75].

\section{Ascendent Identification Scheme (AIS)}

The AIS aims at determining the linear subsystem parameters $\left(a_{i}, b_{i}\right)$ as well as $m$ points located (totally or partly) on the nonlinearity ascendent border. It presents a structural symmetry with the previous DIS scheme and is designed following the same steps. For space limitation, the detailed design procedure is skipped making the focus on the resulting practical identification procedure, emphasizing there the changes with respect to the DIS procedure. It is worthy pointing out that the two schemes are independent i.e. none of them relies on the results of the other. Therefore, it is up to the user to decide which one is applied first.

\subsection{Brief presentation of the AIS}

The AIS procedure follows similar steps as the DIS:
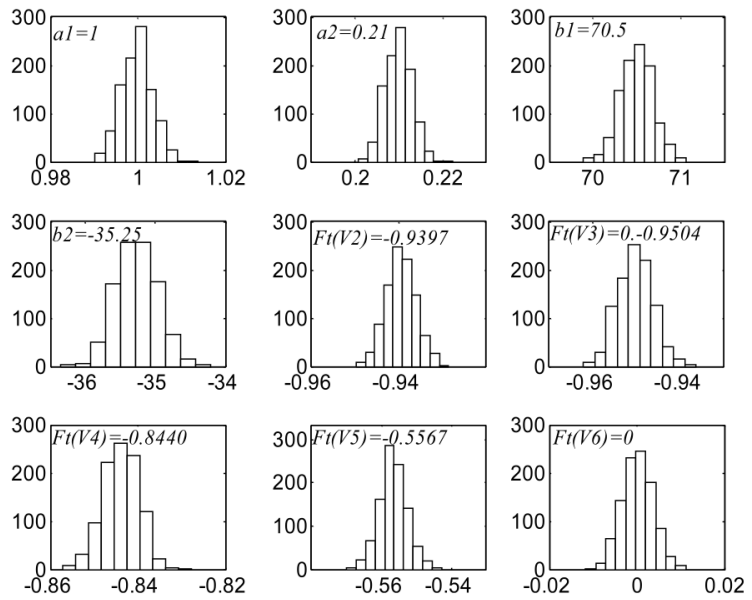

Fig. 12. Histograms of the estimates obtained when running up the DIS scheme 1000 times. The true value of each estimated quantity is indicated in the corresponding histogram. The notation $F t$ refers to $\tilde{F}$.

1. Choose a working interval $\left[\begin{array}{ll}v_{m} & v_{M}\end{array}\right]$ (as in Step 1 of the DIS) and select therein the abscissas $V_{j}^{a}(j=1, \ldots, m)$ of the points to be determined on the ascendent border observing the condition:

$v_{M}=V_{1}^{a}>V_{2}^{a}>\cdots>V_{m}^{a}>v_{m}$.

2. Apply the step input signal $v(t)=v_{0}^{a}(t)$ defined by

$v(t)=v_{0}^{a}(t) \stackrel{\text { def }}{=} \begin{cases}v_{M} & \text { if } t=0 \\ v_{m} & \text { if } t \geq 1\end{cases}$

Compute the mean value $\bar{y}_{0}^{a}$ of the system response, over $a$ sufficiently large observation interval.

3. Apply the periodic pulse sequence $v^{a}(t)$ defined by:

$v^{a}(t)= \begin{cases}V_{j}^{a} & \text { for } t=t_{k}+\tau_{j} ; j=1, \ldots, m \\ v_{m} & \text { otherwise }\end{cases}$

where the $\tau_{j}$ 's are as in Theorem 3.1. Get a sample of the resulting system response $y(t)(t=1, \ldots, N$, for a large $N)$.

4. Construct the following centered signals and vector:

$$
\begin{aligned}
& \tilde{v}(t)=v(t)-v_{m}, \quad \tilde{y}(t)=y(t)-\bar{y}_{0}^{a}, \\
& \tilde{F}^{a}(\tilde{v}(t)) \stackrel{\text { def }}{=} F^{a}(v(t))-u_{m} \quad(t=1, \ldots, N) \\
& \Phi^{a}(t)^{T}=[-\tilde{y}(t-1) \ldots-\tilde{y}(t-n) \\
& \tilde{v}^{a}(t-1) P_{1}^{a}\left(\tilde{v}^{a}(t-1)\right) \ldots \tilde{v}^{a}(t-n) P_{1}^{a}\left(\tilde{v}^{a}(t-n)\right) \\
& \ldots \ldots \\
& \left.\quad \ldots \ldots \tilde{v}^{a}(t-n) P_{m}^{a}\left(\tilde{v}^{a}(t-n)\right)\right] \\
& \tilde{v}_{j}^{a}(t-1) P_{m}^{a}\left(\tilde{v}^{a}(t-1)\right) \stackrel{\text { def }}{=} \prod_{\substack{i=1 \\
i \neq j}}^{m} \frac{w-W_{i}^{a}}{W_{j}^{a}-W_{i}^{a}} ; \quad W_{j}^{a} \stackrel{\text { def }}{=} V_{j}^{a}-v_{m} \\
& \quad(j=1, \ldots, m) .
\end{aligned}
$$

5. Compute the estimates $\hat{\Theta}^{a}(N), \hat{c}_{j}^{a}(N)$ and $\hat{F}^{a}\left(V_{j}^{a}, N\right)(j=1, \ldots$, $m$ ) replacing in the estimators (3.21), (3.22b) and (3.23) the upper script ' $d$ ' by ' $a$ '. Compute the estimates $\hat{b}_{i}(N)(i=1, \ldots, n)$ using the estimator: $\hat{b}_{i}(N)=\hat{\mu}_{i 1}(N) W_{1}^{a}$.

6. End of the identification procedure. 


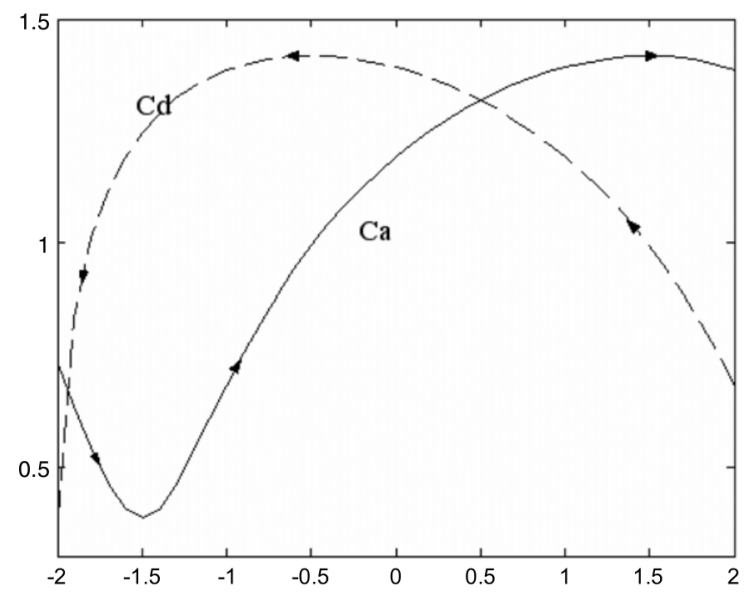

Fig. 13. Lateral borders of the considered switch nonlinearity.

Using similar arguments as in Theorem 3.1, one gets the consistency results formalized in the next statement.

Theorem 4.1. Consider the system (2.1), subject to Assumptions 1 and 2 and excited by the signal $\tilde{v}^{a}(t)$ defined by (4.2). Let the period $T$ and the indexes $\tau_{j}$ in (4.2) be as in Theorem 3.1. Then one has:

(1) The vector sequence $\Phi^{a}(t)$ defined by (4.4) possesses the weak persistent excitation property i.e. $\lim _{N \rightarrow \infty} \frac{1}{N} \sum_{i=1}^{N} \Phi^{a}(t) \Phi^{a}(t)^{T}$ $>\beta$ I w.p. 1 , for some $\beta>0$.

(2) All estimators are consistent i.e. $\hat{\Theta}^{a}(N) \rightarrow \Theta^{a}, \hat{b}_{i}(N) \rightarrow b_{i}$, $\hat{c}_{j}^{a}(N) \rightarrow c_{j}^{a}, \hat{F}^{a}\left(V_{j}^{a}, N\right) \rightarrow F^{a}\left(V_{j}^{a}\right)$ as $N \rightarrow \infty$ (w.p.1).

\subsection{AIS simulation}

The AIS is illustrated considering a Hammerstein-like system with a switch nonlinearity $F[$.$] . The linear subsystem is initially$ (before scaling) characterized by:

$A_{\text {init }}\left(q^{-1}\right)=1-1.25 q^{-1}+0.375 q^{-2}$ and

$B_{\text {init }}\left(q^{-1}\right)=4 q^{-1}-q^{-2}$.

The switch nonlinearity is initially characterized by the following lateral curves:

$C_{\text {init }}^{a}(v)=\log \left(-4 v^{3}-27 v-30\right)$;

$C_{\text {init }}^{d}(v)=\log \left(4 v^{3}-12 v^{2}-15 v+53\right)$

where $\log ($.) denotes the natural logarithm function. The error $\xi(t)$ in (2.1) is presently chosen to be a band-limited white-noise with variance 0.15 . The effect of $\xi(t)$ on the output $y(t)$ is amplified by $1 / A\left(q^{-1}\right)$ whose static gain is 8 . The working interval is $\left[v_{m} v_{M}\right]=$ $\left[\begin{array}{ll}-2 & 2\end{array}\right]$ implying following relations: $u_{m}=C_{\text {init }}^{d}\left(v_{m}\right)=1.0986$, $u_{M}=C_{\text {init }}^{a}\left(v_{M}\right)=3.9512$ and $K=u_{M}-u_{m}=2.8526$. Given model multiplicity, the focus is made on the unique model that satisfies the property (3.11c). The corresponding linear subsystem is characterized by the polynomials:

$$
\begin{aligned}
A\left(q^{-1}\right) & =A_{\text {init }}\left(q^{-1}\right)=1-1.25 q^{-1}+0.375 q^{-2} ; \\
B\left(q^{-1}\right) & =K B_{\text {init }}\left(q^{-1}\right)=K\left(4 q^{-1}-q^{-2}\right) \\
& =11.4105 q^{-1}-2.8526 q^{-2} .
\end{aligned}
$$

The new nonlinearity is a switch type bordered by the following curves (Fig. 13):

$$
\begin{aligned}
& C_{a}(v)=\frac{C_{\text {init }}^{a}(v)}{K}=0.3506 \log \left(-4 v^{3}+27 v+30\right) \\
& C_{d}(v)=\frac{C_{\text {init }}^{d}(v)}{K}=0.3506 \log \left(4 v^{3}-12 v^{2}-15 v+53\right) .
\end{aligned}
$$
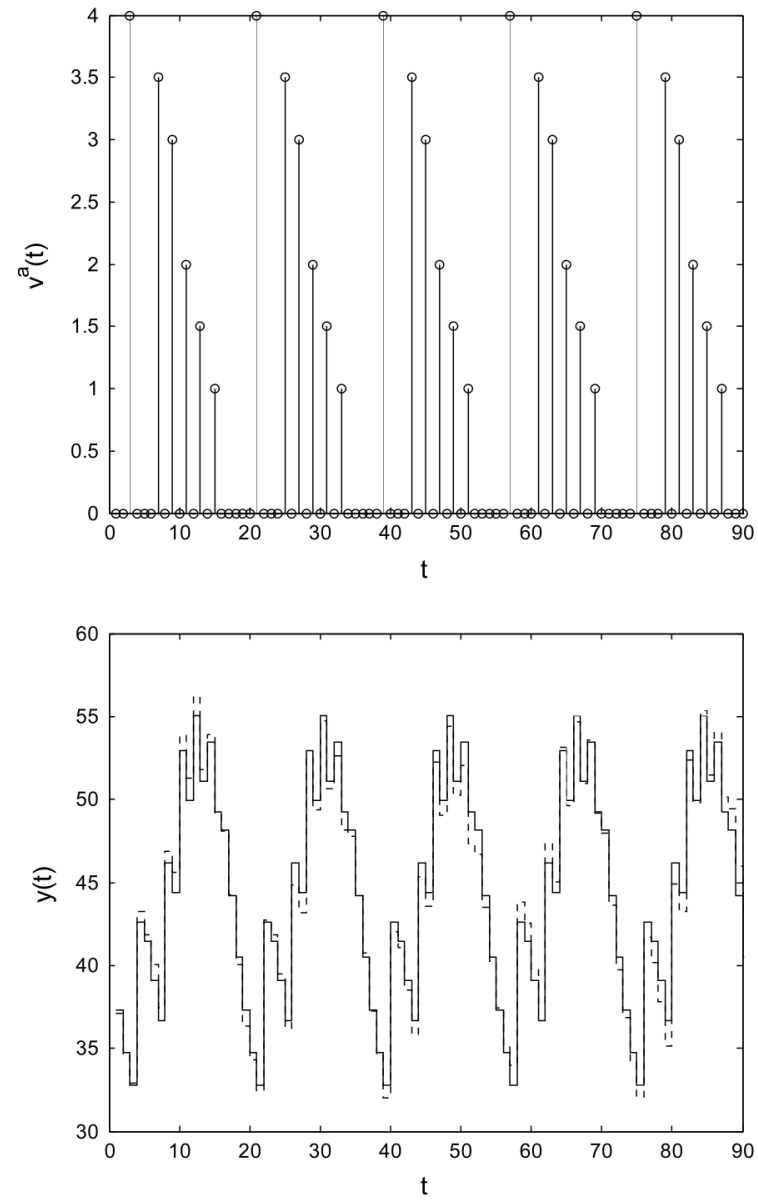

Fig. 14. Signals obtained over five periods in the AP-experiment. Above: input signal $v^{a}(t)$ defined by (4.1). Below: system output $y(t)$ (solid) and output of the model induced by the estimate $\hat{\Theta}^{a}$ (dashed). For clarity, the output signals are represented by their zero-order hold versions.

The identification objective is to estimate the linear subsystem parameters as well as $m=6$ points of the nonlinearity ascendent border. Let us focus on the points of abscissas:

$v_{M}=V_{1}^{a}=2 ; \quad V_{2}^{a}=1.5 ; \quad V_{3}^{a}=1 ; \quad V_{4}^{a}=0 ;$

$V_{5}^{a}=-0.5 ; \quad V_{6}^{a}=-1$.

Following the AIS procedure (Section 4.1), the step input (4.1) is first applied and the mean value (over a sufficiently large interval $[0 \mathrm{~L}]$ ) of the resulting system response is computed. This presently yields (with $L=2000$ ) the estimates $\hat{\bar{y}}_{0}^{a}=26.37$ of the (unknown) true output mean value $\bar{y}_{0}^{a}$. Then, the periodic pulse signal (4.2) is applied over the interval [0 N], with $N=8000$. This is represented, together with the resulting response $y(t)$, by Fig. 14 . These input-output data, $\left(v^{a}(t), y(t)\right)$, are then centered according to (4.3) and used to construct the regressor sequence $\Phi^{a}(t)$ defined by (4.4). The latter is then based upon to estimate $\Theta^{a}$ using the least squares estimator (3.21) replacing there the upper script ' $d$ ' by ' $a$ '. The obtained estimate is:

$$
\begin{aligned}
\hat{\Theta}^{a}= & {\left[\begin{array}{llllll}
-1.2517 & 0.3764 & 2.8500 & -0.7195 & 3.3656 \\
& -0.8502 & 3.8295 & -0.9672 & 4.6098 & -1.1602 \\
& 4.6213 & -1.1487 & 3.3877 & -0.8719
\end{array}\right] . }
\end{aligned}
$$

Finally, using the estimators (3.22a) to (3.23), where the upper script ' $d$ ' is replaced by ' $a$ ', we get the remaining estimates. Tables 4 and 5 show that the obtained estimates are quite close to their true values. This is further illustrated by Fig. 14 which shows the outputs of the true system and the estimated model. Finally it is seen 


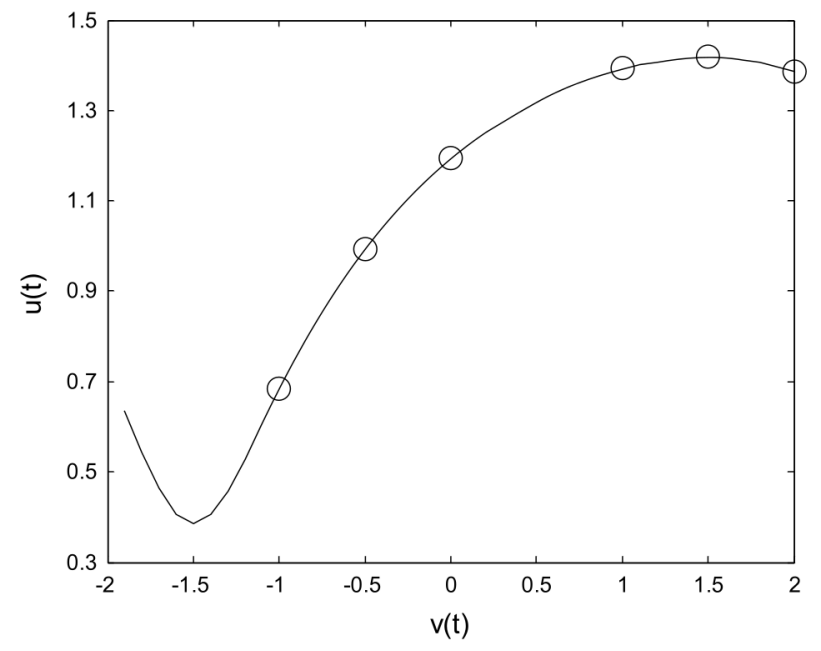

Fig. 15. Nonlinearity ascendent border. The circles indicate the identified points $\left(V_{j}^{a}, \hat{F}^{a}\left(V_{j}^{a}\right)\right)$.

from Fig. 15 that all estimates of the points $\left(V_{j}^{a}, F^{a}\left(V_{j}^{a}\right)\right)$ are actually located on the true nonlinearity ascendent border.

Remark 3. (a) Note that, in Step 5 of the procedure AIS, the estimates $\hat{b}_{i}(N)(i=1 \ldots n)$ are not obtained from the estimator (3.22a) substituting there the subscript ' $a$ ' to ' $d$ ' (as this is the case for the other estimated quantities in Step 5). The reason is that the model focused on presently must be the same as the one focused on in Section 3. But, this model is characterized by (3.11c) which implies the following relations:

$$
\begin{aligned}
& \tilde{F}^{a}\left(W_{1}^{a}\right)=1, \quad c_{1}^{a}=\frac{1}{W_{1}^{a}}, \quad b_{i}=\frac{\mu_{i 1}^{a}}{c_{1}^{a}}=\mu_{i 1}^{a} W_{1}^{a} \\
& \quad(i=1, \ldots, n) .
\end{aligned}
$$

It is the last relation that suggests the estimator used in Step 4 of AIS.

(b) An immediate consequence of the symmetry that exists between the AIS and DIS is that the linear subsystem parameters are estimated by both schemes. This may be a quite interesting feature in practical applications. Indeed, in case the true system nonlinearity is not what it is supposed to be (i.e. switch or backlash), the two schemes will not yield the same parameter estimates for the linear subsystem. Then, one has to look for an alternative identification method.

(c) In those practical situations where there is no doubt on the switch/backlash nature of the system nonlinearity then, provided that the linear subsystem is minimum phase, the AIS may be simplified so that this subsystem is not estimated again. Actually, if the system is minimum phase, the internal signal $u=F[v]$ can be recovered (up to noise) from the output $y(t)$. Roughly, Eq. (2.1) suggests the following estimator to get $u=F[v]$ :

$\hat{B}\left(q^{-1}\right) \hat{u}(t) \approx \hat{A}\left(q^{-1}\right) y(t)$

where $\hat{A}\left(q^{-1}\right) \approx A\left(q^{-1}\right)$ and $\hat{B}\left(q^{-1}\right) \approx B\left(q^{-1}\right)$ are the estimates provided by the DIS (in Section 3 ). Then, the ascendent border is easily identified, based on the relation $\hat{u} \approx F[v]$, using an input signal $v(t)$ like (4.2) that makes the operating point $(v(t), \hat{u}(t))$ only move on that border.

\section{Conclusion}

A new identification approach has been developed to cope with Hammerstein-like systems that include dynamic nonlinearities
Table 4

Parameters of linear subsystem characterized by (4.6) and their estimates.

\begin{tabular}{llllr}
\hline$j$ & $a_{j}$ & $\hat{a}_{j}$ & \multicolumn{1}{c}{$b_{j}$} & $\hat{b}_{j}$ \\
\hline 1 & -1.25 & -1.2579 & 11.4105 & 11.4146 \\
2 & 0.375 & 0.3813 & -2.8526 & -2.8528 \\
\hline
\end{tabular}

Table 5

Parameters of the nonlinearity characterized by (4.7) and their estimates.

\begin{tabular}{lllllll}
\hline$V_{j}^{a}$ & 4 & 3 & 2 & 1 & -1 & -2 \\
\hline$F^{a}\left(V_{j}^{a}\right)$ & 1.3851 & 1.4173 & 1.3918 & 1.1923 & 0.9932 & 0.6821 \\
$\hat{F}^{a}\left(V_{j}^{a}\right)$ & 1.3852 & 1.4170 & 1.3873 & 1.1900 & 0.9939 & 0.6808 \\
\hline
\end{tabular}

of the switch or backlash types. The originality lies in the fact that the nonlinearity borders are not parametric and so are not necessarily straight lines. Interestingly, the borders are allowed to be nonsmooth and noninvertible. The proposed approach consists of two independent and perfectly symmetric schemes. The first one, the DIS presented in Section 3, estimates the linear subsystem parameters as well as $m$ points (totally or partly located) on the descendent border. The second scheme, the AIS presented in Section 4, estimates the linear subsystem parameters as well as $m$ points (totally or partly located) on the ascendent border. Experiment design is a key feature in the design of these identification schemes. The experiments are conceived so that the initial identification problem, involving a dynamic and nonparametric nonlinearity, is decomposed into two simpler problems characterized by parametric static nonlinearities. The full parameterization of the identification problem made possible the construction of linearly parameterized representations, namely (3.19a) and the corresponding one in the AIS (obtained by just replacing upper script ' $d$ ' by ' $a$ '). The generated signals are also shown (Theorems 3.1 and 4.1) to be persistently exciting, ensuring the consistency of all involved estimators.

\section{Appendix. Proof of Theorem 3.1 (Part 1)}

From (3.24b), it follows that $Z^{d}(t)$ can be decomposed as follows:

$Z^{d}(t)^{T}=\left[\begin{array}{ll}Z_{1}(t)^{T} & Z_{2}(t)^{T} \ldots Z_{m}(t)^{T}\end{array}\right]^{T}$

with:

$$
\begin{aligned}
Z_{1}(t)^{T}= & {\left[-z^{d}(t-1) \ldots-z^{d}(t-n)\right.} \\
& \left.\tilde{v}^{d}(t-1) P_{1}^{d}\left(\tilde{v}^{d}(t-1)\right) \ldots \tilde{v}^{d}(t-n) P_{1}^{d}\left(\tilde{v}^{d}(t-n)\right)\right] \\
Z_{j}(t)^{T}= & {\left[\tilde{v}^{d}(t-1) P_{j}^{d}\left(\tilde{v}^{d}(t-1)\right)\right.} \\
& \left.\ldots \tilde{v}^{d}(t-n) P_{j}^{d}\left(\tilde{v}^{d}(t-n)\right)\right] .
\end{aligned}
$$

The rest of the proof is divided in three steps. First, we show that the vector sequence $Z_{1}(t)$ possesses the persistent excitation (PE) property. In step 2 , it is shown that the vector sequences $Z_{j}(t)(j=2 \ldots m)$ share in turn such property. Finally, it will be demonstrated that the global vector $Z^{d}(t)$ satisfies the PE property as stated in Part 1 of Theorem 3.1.

Step 1: Proof that $Z_{1}(t)$ is persistently exciting $(P E)$

Let us show that, there a positive real $\varepsilon_{0}$, such for any integer $k$ :

$\sum_{\tau=1}^{4 n} Z_{1}\left(t_{k}+\tau\right) Z_{1}\left(t_{k}+\tau\right)^{T}>\varepsilon_{0} I_{2 n}$

( $I_{2 n}$ is the $2 n \times 2 n$ identity matrix).

From (3.13) one has, for $k=1,2,3, \ldots$ and $t_{k}-n<t<t_{k}+4 n$ :

$\tilde{v}^{d}(t)= \begin{cases}W_{1}^{d} & \text { for } t=t_{k}+\tau_{1} \\ 0 & \text { otherwise. }\end{cases}$ 
Then, it follows from (3.13) and (3.15):

$P_{j}^{d}\left(\tilde{v}^{d}(t)\right)=0$

for $j=2 \ldots m$ and $t_{k}-n<t<t_{k}+4 n$.

Then, Eq. (3.14a) reduces in the time interval $t_{k}-n<t<t_{k}+4 n$ to:

$P^{d}\left(\tilde{v}^{d}(t)\right)=c_{1}^{d} \tilde{v}^{d}(t) P_{1}^{d}\left(\tilde{v}^{d}(t)\right)$.

In view of (A.6), Eq. (3.24a) in turn reduces to:

$A\left(q^{-1}\right) z^{d}(t)=c_{1}^{d} B\left(q^{-1}\right) \tilde{v}^{d}(t) P_{1}^{d}\left(\tilde{v}^{d}(t)\right)$

for $t_{k}-n<t<t_{k}+4 n$ and $k=1,2,3, \ldots$.

In view of (3.18) and (A.2), Eq. (A.8) can also be given the following regressive form:

$z^{d}(t)=Z_{1}(t)^{T} \Theta_{1}^{d}$

for $t_{k}-n<t<t_{k}+4 n$ and $k=1,2,3, \ldots$

$\Theta_{1}^{d}=\left[a_{1} \ldots a_{n} \mu_{11}^{d} \ldots \mu_{n 1}^{d}\right]^{T}$.

Applying the Technical Excitation Lemma (Giri et al., 2002) to (A.8) it follows from (A.5) and (A.9) that the regression vector $Z_{1}(t)$, defined by (A.2), is persistently exciting in the sense of (A.4).

Step 2: Proof that $Z_{j}(t)(j=2 \ldots m)$ are $P E$

Let us demonstrate that, for all $k$ :

$\sum_{\tau=1}^{n} Z_{j}\left(t_{k}+n(j+2)+\tau\right) Z_{j}\left(t_{k}+n(j+2)+\tau\right)^{T} \geq \varepsilon_{1} . I_{n}$

for $j=2, \ldots, m$

where $\varepsilon_{1}=\inf _{j=2, \ldots, m}\left(W_{j}^{d}\right)^{2}$ and $I_{n}$ denotes the $n \times n$ identity matrix.

Using (3.13) and (3.15), it follows from (A.3) that, for any $j=$ $2, \ldots, m ; k=1,2,3, \ldots ; \tau=1, \ldots, n$ :

$Z_{j}\left(t_{k}+n(j+2)+\tau\right) Z_{j}\left(t_{k}+n(j+2)+\tau\right)^{T}$

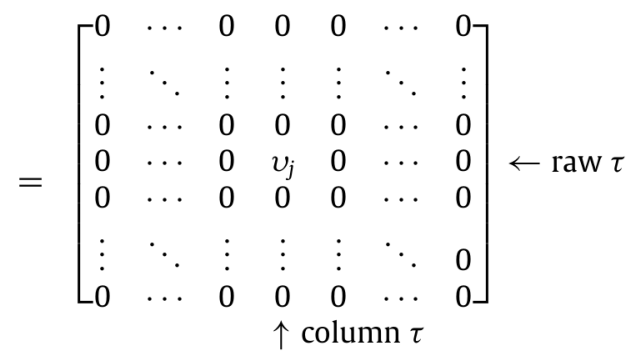

with

$v_{j} \stackrel{\text { def }}{=} W_{j}^{d} P_{j}^{d}\left(W_{j}^{d}\right)=W_{j}^{d}$.

(A.11) clearly follows from (A.12a)-(A.12b).

Step 3: Proof that $Z^{d}(t)$ is $P E$

Let us now demonstrate, by contradiction, that $Z^{d}(t)$ is PE in the sense of Part 1 of Theorem 3.1. To this end, suppose that $Z^{d}(t)$ is not $P E$. Then for any sequence of positive real numbers $\{\varepsilon(i)\}$ such that:

$\lim _{i \rightarrow \infty} \varepsilon(i)=0$

there exists a unit vector sequence $\{X(i)\}$ (i.e. $\|X(i)\|=1$ ) and a subsequence $t_{k_{i}}$ such that for all $i$ :

$\max _{0 \leq \tau \leq n(3+N)}\left|Z^{d}\left(t_{k_{i}}+\tau\right)^{T} X(i)\right| \leq \varepsilon(i)$.

Following the partition (A.1), X(i) can in turn be decomposed as follows:

$X(i)^{T}=\left[\begin{array}{lll}x_{1}(i)^{T} & x_{2}(i)^{T} \ldots x_{m}(i)^{T}\end{array}\right]^{T}$ where the subvector $x_{j}(i)$ is of the same dimension as $Z_{j}^{d}$. On the other hand, using (3.13), (3.15), (A.1)-(A.3) and (A.15), it follows that, for any integer numbers $k$ and $i$ :

$$
\begin{aligned}
& Z^{d}\left(t_{k}+\tau\right)^{T} X(i)=Z_{1}^{d}\left(t_{k}+\tau\right)^{T} x_{1}(i) \\
& \text { for } \tau=1, \ldots, 4 n \\
& Z^{d}\left(t_{k}+n(j+2)+\tau\right)^{T} X(i)=Z_{1}^{d}\left(t_{k}+n(j+2)+\tau\right)^{T} x_{1}(i) \\
& \quad+Z_{j}^{d}\left(t_{k}+n(j+3)+\tau\right) x_{j}(i) \\
& \quad \text { for } j=2, \ldots, m ; \tau=1, \ldots, n .
\end{aligned}
$$

Using (A.4), one gets from (A.16) that, for all $k$ and all $i$ :

$\left\|x_{1}(i)\right\|^{2}<\frac{4 n}{\varepsilon_{0}} \max _{1 \leq \tau \leq 4 n}\left|Z^{d}\left(t_{k}+\tau\right)^{T} X(i)\right|^{2}$

which, together with (A.13)-(A.14), implies that:

$\lim _{i \rightarrow \infty}\left\|x_{1}(i)\right\|^{2}=0$

Similarly, using (A.11), one gets from (A.17) that for all integers $k$, $i$ and $j=2, \ldots, m$ :

$$
\begin{aligned}
\left\|x_{j}(i)\right\|^{2}<n & \left(\frac{\max _{1 \leq \tau \leq n}\left\|Z^{d}\left(t_{k}+n(j+2)+\tau\right)^{T} X(i)\right\|^{2}}{\varepsilon_{1}}\right. \\
& \left.+\frac{\max _{1 \leq \tau \leq n}\left\|Z_{1}\left(t_{k}+n(j+2)+\tau\right)^{T} x_{1}(i)\right\|^{2}}{\varepsilon_{1}}\right) .
\end{aligned}
$$

Using (A.14),(A.18) and the fact that the sequence $Z_{1}(t)$ is bounded, it follows from (A.19) that:

$\lim _{i \rightarrow \infty}\left\|x_{j}(i)\right\|=0$ for $j=2, \ldots, m$.

Combining (A.18) and (A.19), one gets from (A.20) that $\lim _{i \rightarrow \infty}\|X(i)\|=0$ which contradicts the fact that $X(i)$ is unit vector. Hence, Part 1 of Theorem 3.1 is proved.

\section{References}

Bai, E. W. (2002). Identification of systems with hard input nonlinearities of unknown structure. Automatica, 38, 853-860.

Bai, E. W. (2002). Identification of linear systems with hard input nonlinearities of known structure. Automatica, 38, 853-860.

Bai, E. W., \& Li, D. (2004). Convergence of the iterative Hammerstein system identification algorithm. IEEE Transactions on Automatic Control, 49(11), 1929-1940.

Cerone, V, \& Regruto, D. (2007). Bounding the parameters of linear systems with input backlash. IEEE Transactions on Automatic Control, 52, 531-536.

Chaoui, F. Z., Giri, F., Rochdi, Y., Haloua, M., \& Naitali, A. (2005). System Identification based on Hammerstein model. International Journal of Control, 78(6), 430-442.

Eskinat, E., Johnson, S., \& Luyben, W. L. (1991). Use of Hammerstein models in identification of nonlinear systems. AIChE Journal, 37(2), 255-268.

Giri, F., Chaoui, F. Z., \& Rochdi, Y. (2004). Recursive identification of systems with hard input nonlinearities of known structure. In American control conference. Boston, MA, USA (pp. 4764-4769).

Giri, F., Chaoui, F. Z., \& Rochdi, Y. (2001). Parameter identification of a class of Hammerstein plants. Automatica, 37, 749-756.

Giri, F., Chaoui, F. Z., \& Rochdi, Y. (2002). Interval excitation through impulse sequences. A technical lemma. Automatica, 38, 457-465.

Giri, F., Rochdi, Y., Chaoui, F. Z., \& Brouri, A. (2008). Identification of Hammerstein systems in presence of hysteresis-backlash and hysteresis-relay nonlinearities. Automatica, 44, 767-775.

Giri, F., Rochdi, Y., Elayan, E., Brouri, A., \& Chaoui, F.Z. (2008). Hammerstein systems identification in presence of hysteresis-backlash nonlinearity. In IFAC world congress. Seoul (pp. 7859-7864).

Greblicki, W., \& Pawlak, M. (2008). Nonparametric system identification. Cambridge University Press. 
Greblicki, W., \& Pawlak, M. (1994). Nonparametric recovering nonlinearities in block oriented systems with the help of Laguerre polynomials. Control-Theory and Advanced Technology, 10(part 1), 771-791.

Gu, X., Bao, Y., \& Lang, Z. (1988). A parameter identification method for a class of discrete-time nonlinear systems. In Proceedings of the IMACS world congress, Paris: Vol. 4 (pp. 627-629).

Hammerstein, A. (1930). Nichtlineare integralgleichung nebst anwendungen. Acta Mathematica, 54, 117-176.

Hasiewicz, Z., \& Mzyk, G. (2009). Hammerstein system identification by nonparametric instrumental variables. International Journal of Control, 82(3), 440-455.

Hasiewicz, Z., Sliwinski, P., \& Mzyk, G. (2008). Nonlinear system identification under various prior knowledge. In IFAC world congress. Seoul (pp. 7849-7857)

Kim, J., \& Konstantinou, K. (2001). Digital predistortion of wideband signals based on power amplifier model with memory. IEE Electronics Letters, 37(23), 1417-1418.

Narendra, K. S. \& Gallman, P. G. (1966). An iterative method for the identification of nonlinear systems using a Hammerstein model. IEEE Transactions on Automatic Control, 11(7), 546-550.

Palanthandalam-Madapusi, H. J., Bernstein, D. S., \& Ridley, A. J. (2006). Subspace identification of periodically switching Hammerstein-Wiener models for magnetospheric dynamics. In 14th IFAC symposium on system identification. Newcastle, Australia (pp. 535-540).

Phillips, G. M. (2003). Interpolation and approximation by polynomials. Springer.

Stoica, P. (1981). On the convergence of an iterative algorithm used for Hammerstein system identification. IEEE Transactions on Automatic Control, 26(4), 967-969.

Stoica, P., \& Söderström, T. (1982). Instrumental-variable methods for identification of Hammerstein systems. International Journal of Control, 35(3), 459-476.

Voros, J. (1997). Parameter identification of discontinuous Hammerstein systems. Automatica, 33, 1141-1145 